Geophysical and Astrophysical Fluid Dynamics

Vol. 00, No. 00, 00 Month 20xx, 1-24

\title{
The interaction of two asymmetric quasi-geostrophic vortex patches
}

\author{
M.M. Jalalił \& D.G. Dritschel †* \\ (Received 00 Month 20xx; final version received 00 Month 20xx)
}

\begin{abstract}
Herein we study the general interaction of two vortex patches in a single-layer quasi-geostrophic shallowwater flow. Steadily-rotating equilibrium states are found over a wide parameter space spanning the Rossby deformation length, vortex area ratio, potential vorticity ratio, and gap between their innermost edges. A linear stability analysis is then used to identify the critical gap separating stable and unstable solutions, over the entire range of area and potential vorticity ratios, and for selected values of the Rossby deformation length. A representative set of marginally unstable equilibrium states are then slightly perturbed and evolved by an accurate contour dynamics numerical algorithm to understand the long-term fate of the instabilities. Not all instabilities lead to vortex merger; many in fact are characterised by weak filamentation and a small adjustment of the vortex shapes, without merger. Stronger instabilities lead to material being torn from one vortex and either wrapped around the other or reduced to ever thinning filamentary debris. A portion of the vortex may survive, or it may be completely strained out by the other.
\end{abstract}

Keywords: Quasi-geostrophy, vortex patches, contour dynamics

\section{Introduction}

The study of fundamental aspects of vortex dynamics has a long history dating back to Helmholtz, Kelvin and Kirchhoff in the 19th century (von Helmholtz 1858, Thomson 1869, Kirchhoff 1876); (see also Lamb 1932, Moffatt 2008, Aref 2010). The inherently nonlinear character of vortex motion has made its study both challenging and fascinating. The greatest progress has been made in two-dimensional incompressible fluid flows in which vertical variations are neglected. Then, in the absence of viscosity and external forces, the vortex dynamics reduces to material conservation of the vertical component of vorticity together with linear but non-local 'inversion relations' determining the fluid motion from the instantaneous distribution of vorticity. This seemingly simple situation is nonetheless capable of producing exceedingly complex fluid motion and surprisingly rich behaviour, as evidenced for example in two-dimensional turbulence (cf. Burgess et al. (2017) and references therein).

In geophysical flows like the atmosphere, oceans and other planetary atmospheres, the effects of rotation and stratification combine to render fluid motion 'layerwise two-dimensional' over a wide range of scales (Vallis 2006). These effects result in a leading-order balance between the Coriolis acceleration and the horizontal pressure gradient, and between the gravitational acceleration and the vertical pressure gradient, respectively known as 'geostrophic' and 'hydrostatic' balance. Fluid motion is then dominated by the slow, ponderous advection of 'potential vorticity' (PV), a scalar field intimately related to the circulation on isentropic (constant entropy) or isopycnal (constant density) surfaces (Hoskins et al. 1985). The relatively fast inertia-gravity waves and acoustic waves often contribute only weakly to the fluid dynamics (Ford et al. 2000, Mohebalhojeh and Dritschel 2001, 2004, McKiver and Dritschel 2008) and are filtered by geostrophic and hydrostatic balance. Moreover, neglecting viscosity

*Corresponding author. Email: david. dritschel@st-andrews.ac.uk 
and diabatic effects, PV is materially conserved (like circulation) on material surfaces which are approximately horizontal.

This state of affairs can give rise to long-lived vortices, such as Jupiter's Great Red Spot (Williams 1978, Ingersoll et al. 2007, Read 2011), the Earth's stratospheric polar vortex (McIntyre and Palmer 1983, Dunkerton and Delisi 1986, McIntyre 1995), and the multitude of vortices observed by satellites in every ocean basin and sea (Carton 2001, Beron-Vera et al. 2013). To help better understand these observations, over the past forty years a considerable effort has gone into developing simplified models of geophysical flows. These range from single-layer, two-dimensional ('barotropic') models, to quasi-geostrophic and semi-geostrophic shallow water models, to multi-layer quasi-geostrophic and continuously stratified models (see Pedlosky (1979), Polvani (1988), Polvani et al. (1989), Waugh and Dritschel (1991), Kizner et al. (2008), Carton (2010), Samelson et al. (2014) for a sample of the vast literature).

Of the various available models, the quasi-geostrophic shallow water (QGSW) model is the simplest one capturing the effects of both rotation and stratification in a single fluid layer (Płotka and Dritschel 2012, 2013). This model is remarkably similar to the classical twodimensional flow model discussed at the outset, with the only difference being the relation between streamfunction $\psi$ and the circulation invariant $q$, in this case the potential vorticity. Instead of $\nabla^{2} \psi=q$, in the QGSW model $\nabla^{2} \psi-\gamma^{2} \psi=q$ holds, where $\gamma$ is the inverse of the 'Rossby deformation length' $L_{D}$. This length is given by $\sqrt{g H} / f$, where $g$ is the acceleration due to gravity (or 'reduced' gravity when considering a fluid layer lying on top of another much deeper layer at rest), $H$ is the mean depth of the layer, and $f$ is the Coriolis frequency. As such, $L_{D}$ incorporates both the effects of buoyancy and rotation. When $L_{D} \rightarrow \infty$, we recover the two-dimensional incompressible model. The effect of $L_{D}$ is to greatly weaken long-range interactions over distances $r>L_{D}$.

In geophysical fluid dynamics, the QGSW model has frequently been employed to study fundamental processes like vortex interactions, jet formation, meandering of oceanic fronts and eddy detachment (Robinson et al. 1975). By filtering fast inertia-gravity waves, the QGSW model allows one to study such processes both efficiently and comprehensively. In regimes relevant to the Earth's atmosphere and oceans, often inertia-gravity waves are of secondary importance and contribute little to the fluid motion; moreover they tend to be weak energetically. In such regimes, relatively slow vortical motions controlled by PV - balanced motions - dominate (Hoskins et al. 1985). More important are the ageostrophic motions occurring at moderate Rossby and Froude numbers (see $\S 1$ below). These too are neglected in the QGSW model, which assumes both numbers are small compared to unity. Nevertheless, much can still be learned from the QGSW model, including importantly the basic mechanisms controlling observed flow phenomena and their dependence on system parameters. The present study focuses on vortex interactions, and is the first comprehensive study of its kind. The results are perhaps most relevant to near-surface eddy interactions in the oceans, but may also be relevant to the ubiquitous vortex motions seen in the atmospheres of the giant gas planets. The exceptionally wide range of parameters studied may help to explain vortex persistence in natural flows, to delineate typical forms of interaction, and to guide further work using less idealised models.

Some of the earliest numerical methods used to study two-dimensional vortex dynamics were based on Contour Dynamics (CD). Zabusky et al. (1979) introduced CD to study the evolution of piecewise-constant vorticity distributions, or 'vortex patches'. For vortex patches, the flow evolution depends entirely on the shapes of their boundaries or 'contours'. These contours move and distort in a way which can be computed from contour integrals around their boundaries (details may be found in $\S 2$ below). CD represented a major advance in the study of vortex dynamics, effectively reducing two space dimensions to one, represented by a single coordinate such as arc length. This permitted the study of vortex equilibria, their stability and their nonlinear dynamics without the effect of diffusion, numerical or real. 
More than a century after Kirchhoff's discovery of the steadily-rotating elliptical vortex patch, Deem and Zabusky (1978a,b) discovered several new families of (relative) equilibria, 'V-states', using an iterative numerical method based on CD. These families bifurcate from the circular vortex patch as $m$-fold symmetric waves, with $m=2$ corresponding to Kirchhoff's elliptical vortex. At large amplitude, these families end in 'corner' states (except for $m=2$ ), patches with right-angled corners coinciding with flow stagnation points. Zabusky and coworkers (Zabusky et al. 1979, Overman and Zabusky 1982, Wu et al. 1984) further investigated the nonlinear interaction of vortex patches using CD. Zabusky et al. (1979) were the first to identify the critical merger distance for two initially circular vortex patches, of identical area and vorticity (this is approximately 3.4 vortex radii). Saffman and Szeto (1980) computed the equilibrium states for two identical vortex patches and conjectured that they are linearly unstable at an extremum in angular momentum or energy (see $\S 3$ below). This instability was computed explicitly by Dritschel (1985), who also computed the equilibrium states for $m>2$ identical vortex patches equally-spaced on a ring. Overman and Zabusky (1982) and Dritschel (1986) confirmed using CD that the linear instability leads to vortex merger in the nonlinear dynamics. Steadily-translating pairs of equal-sized, opposite-signed vortices were obtained numerically by Pierrehumbert (1980) and Wu et al. (1984) but their linear stability was not investigated.

Dritschel and Waugh (1992) were the first to comprehensively examine the interaction between two vortices of different sizes but of equal vorticity. Starting from circular vortex patches, they showed that vortex merger is exceptional, occurring only for vortices of nearly equal size. Otherwise, the vortices may not merge at all but instead exchange material and expel thin filaments, or the smaller vortex may be completely strained out. Their simulations were made possible by a major extension to CD called 'Contour Surgery' (CS) developed by Dritschel (1988) (see also the extended review in Dritschel (1989a)). CS allowed for much longer simulations by limiting the smallest scales which could develop in the flow. This was done by automatically removing very thin filaments, separating parts of a vortex patch joined by a thin filament into two, or by joining two patches separated by a narrow gap. While this changes the topology of the flow, it has only a weak impact on the velocity field due to the small circulation changes involved. In particular, the main features of the flow, including many details, are reproduced for widely varying scales of surgery over moderate integration times (Dritschel 2005).

Dritschel (1995) extended the study of Dritschel and Waugh (1992) by determining the equilibrium shapes and stability of two unequal-sized vortices, for both equal and opposite vorticity. A linear stability analysis confirmed that an extremum in angular momentum or energy coincides with the margin of stability for like-signed vortices, but not for oppositesigned ones. CS simulations were used to explore the long-term evolution of unstable equilibria, confirming that vortex merger is exceptional: as little as a $1 \%$ difference in vortex area leads to partial merger, an asymmetric two-vortex state where one vortex is larger than the other. Unstable opposite-signed equilibria were found to break into three or more vortices, two of which form a less tightly-bound dipole leaving the others behind. Dritschel and Zabusky (1996) examined dilute two-dimensional turbulence on a sphere and confirmed that strong vortex interactions more often lead to the creation of more vortices rather than the merger into one.

Yasuda and Flierl (1995), Trieling et al. (2005) and Brandt and Nomura (2010) generalised the study of Dritschel and Waugh (1992) to consider unequal and (in the latter two studies) non-uniform vorticity, to compare with laboratory experiments at moderate Reynolds numbers. Unequal vorticity and size creates a competition: a small but intense vortex can dominate a large but weak vortex. The small vortex typically burrows to the centre of the larger vortex, while generating significant filamentary debris in the process. The effect of relatively weak vorticity in the vortex periphery was shown to favour complete vortex merger 
(see also the earlier study by Melander et al. (1987)). By contrast, sharp-edged vortices were found to exhibit similar regimes of interaction found in Dritschel and Waugh (1992). Such vortices are commonly found at very high Reynolds numbers due to the process of "vortex stripping' caused by the strain field associated with nearby vortices (Dritschel 1989b, Legras and Dritschel 1993, Mariotti et al. 1994, Yao et al. 1995).

Families of singly-connected rotating vortex patch equilibria were discovered by Kamm (1987) bifurcating from the Love instability points (Love 1893) of Kirchhoff's elliptical vortex (see also Hmidi and Mateu (2016)). These families were computed in detail by LuzzattoFegiz and Williamson $(2010,2011)$, who found a variety of states including tear drop-shaped, dumbbell-shaped and many others. However, all of these states are linearly unstable (Dritschel et al. 2018). Opposite-signed vortex patch equilibria having both unequal area and vorticity were obtained by Makarov and Kizner (2011). These states generally rotate about a common point, e.g. by translating on a circular path, except when the vortex circulations are equal and opposite. Then the vortices translate on a straight path.

While there have been many studies of vortex interactions in the two-dimensional incompressible model, far fewer have considered the QGSW model even though it is more relevant to atmospheric and oceanic dynamics. The extra parameter, the Rossby deformation length $L_{D}$, adds significant complexity to a problem with already several parameters: vortex separation, area ratio and PV ratio. The earliest studies were conducted by Polvani (1988), Polvani et al. (1989), Polvani (1991) and Waugh and Dritschel (1991). They considered not only the singlelayer QG model, but the two-layer model having both a barotropic (height-independent) mode and a baroclinic (height-varying) mode (Phillips 1954). The two-layer model combines the twodimensional incompressible model and the single-layer, QGSW model. The layer-depth ratio and PV distribution can be used to control the degree to which one mode dominates the other. The QGSW model is recovered in the limit of zero layer-depth ratio and by concentrating the PV in one layer, i.e. for a thin active upper layer lying on top of a quiescent deep layer. The two-dimensional incompressible model is recovered by taking the PV to be identical in both layers.

Polvani (1988), Polvani et al. (1989), Polvani (1991) obtained equilibrium states for a single vortex patch or two patches of the same area and PV (or opposite PV), either in the same layer or in different layers. They showed that the layer-depth ratio and the length $L_{D}$ may profoundly alter the shapes and properties of the equilibrium states. In particular, when $L_{D}$ is small compared to the vortex radius and in the QGSW limit, two-fold symmetric vortex patches vary considerably from an elliptical shape, and terminate in a dumbbell-shaped vortex attached at a single point (a comprehensive study of the effect of $L_{D}$ can be found in Płotka and Dritschel (2012), including linear stability and nonlinear evolution). Waugh and Dritschel (1991) studied the effect of finite $L_{D}$ and the layer depth ratio on filament stability as well as on vortex merger. In the QGSW model, Waugh (1992) showed that the critical merger distance between two identical initially circular patches decreases with $L_{D}$, limiting to twice the vortex radius (corresponding to touching vortices) when $L_{D} \rightarrow 0$.

Two-vortex equilibrium states in the QGSW model also differ considerably from their twodimensional incompressible counterparts. For small $L_{D}$, each vortex remains nearly circular until almost touching (when the gap between the vortices is comparable to $L_{D}$ ). This is due to the exponential fall-off (or slightly faster) of the Green function associated with the inversion operator $\nabla^{2}-\gamma^{2}$ in the QGSW model $\left(\gamma=1 / L_{D}\right)$. Recently, Dritschel et al. $(2018)$ discovered many additional branches of two-fold equilibria related to those found by LuzzattoFegiz and Williamson $(2010,2011)$ bifurcating from elliptical vortices in the two-dimensional incompressible model. These additional branches are all disconnected, and in particular are not on the branch having the circular vortex at one endpoint.

Makarov et al. (2012) (see also references therein) obtained equilibrium states for oppositesigned vortex patches occupying separate layers in a two-layer QG flow. These 'hetons' gener- 
ally propagate if their horizontal centres are not coincident, similar to dipoles in a single layer (Makarov and Kizner 2011). Makarov et al. (2012) examined their stability in CD simulations and found that large vortices (compared to the Rossby deformation length $L_{D}$ ) are subject to 'baroclinic' (or vertical shear) instability (Vallis 2006). This leads to the break-up of the vortices into multiple hetons or more complex structures.

The present study focuses on the single-layer, QGSW model. To date, no comprehensive examination has yet been carried out over the parameter space spanned by $L_{D}$, the vortex separation, area ratio and PV ratio - even for vortex patches. This is the objective here. We only consider like-signed vortices. Opposite-signed vortices behave in fundamentally different ways and will be the focus of a further study. Here, we obtain equilibrium states and their linear stability over an extensive parameter space. Moreover, we examine the fate of instabilities and, in particular, the quasi-equilibrated long-term flow state. $\S 2$ reviews the QGSW model and the CD/CS model used. $\S 3$ describes the shapes and properties of the vortex patch equilibria, including their linear stability. $\S 4$ illustrates the nonlinear evolution of unstable equilibria in a number of representative cases. Our conclusions are presented in $\S 5$.

\section{The mathematical model and its numerical representation}

\subsection{Quasi-Geostrophic Shallow Water}

We employ perhaps the simplest geophysically relevant model of rotating, stratified geophysical flows, namely the Quasi-Geostrophic Shallow Water (QGSW) model. This commonly-used model approximates the dynamics of a thin rotating layer of fluid with a free surface using hydrostatic balance and assuming that the horizontal fluid velocity $\boldsymbol{u}=(u, v)$ is independent of depth, as in the shallow-water model. The QGSW model goes further by using geostrophic balance to relate the horizontal velocity to height gradients, which assumes that the Rossby number $R o$ is sufficiently small to neglect the horizontal acceleration compared to the Coriolis acceleration. Finally, height variations are assumed to be small compared to the mean fluid depth, which is equivalent to assuming that the Froude number $\mathrm{Fr} \sim R o$, where $\mathrm{Fr}=U / \sqrt{g H}$ and $R o=U / f L$. Here $U$ and $L$ are characteristic horizontal velocity and length scales. More rigorously, the QGSW model requires $F r^{2} \ll R o \ll 1$.

The governing equations consist of a single equation expressing material conservation of QG $\mathrm{PV}$,

$$
\frac{\mathrm{D} q}{\mathrm{D} t}=0
$$

assuming an inviscid adiabatic flow, together with 'inversion relations' providing the velocity field $\boldsymbol{u}$ in terms of $q$,

$$
\nabla^{2} \psi-\gamma^{2} \psi=q, \quad \boldsymbol{u}=\nabla^{\perp} \psi
$$

where $\boldsymbol{\nabla}^{\perp}=(-\partial / \partial y, \partial / \partial x)$ is the skewed gradient. Note that $\boldsymbol{\nabla} \cdot \boldsymbol{u}=0$. Here $\gamma=1 / L_{D}$ is the inverse Rossby deformation length. This is the single parameter appearing in the QGSW model. It combines gravity $g$ (or reduced gravity), the mean fluid depth $H$ and the Coriolis frequency $f$, none of which individually appear in the QGSW equations. Notably, the twodimensional incompressible model is recovered in the limit $\gamma \rightarrow 0$.

For an unbounded flow with a compact distribution of PV (non-zero over a finite region), the total energy (kinetic plus potential)

$$
\mathcal{E}=\frac{1}{2} \iint\left(\|\boldsymbol{u}\|^{2}+\gamma^{2} \psi^{2}\right) \mathrm{d} x \mathrm{~d} y=-\frac{1}{2} \iint q \psi \mathrm{d} x \mathrm{~d} y
$$

is conserved. The final expression follows after integration by parts and using Eq.(2). Moreover 
both linear impulse

$$
\mathcal{I}=\iint q \boldsymbol{x} \mathrm{d} x \mathrm{~d} y
$$

and angular impulse

$$
\mathcal{J}=\iint q\|\boldsymbol{x}\|^{2} \mathrm{~d} x \mathrm{~d} y
$$

are conserved, a consequence of translational and rotational symmetry of the equations. Finally, in general an infinite set of Casimirs

$$
\mathcal{C}=\iint F(q) \mathrm{d} x \mathrm{~d} y
$$

are conserved, where $F$ is an arbitrary convex function. This is a consequence of particlerelabelling symmetry, or simply the material conservation of PV. Note that for piecewiseuniform PV, the number of distinct Casimirs is equal to the number of distinct values of PV. The Casimirs are combinations of the areas of each distinct value of PV, and are conserved on account of incompressibility.

\subsection{Contour dynamics and contour surgery}

Any layerwise two-dimensional flow governed by the advection of a materially-conserved tracer $q$, and whose velocity field $\boldsymbol{u}$ may be recovered by linear inversion relations from $q$, admits a contour dynamics (CD) formulation (Dritschel 1989a, Waugh and Dritschel 1991, Dritschel and Saravanan 1994). In CD, the distribution of $q$ is represented by a set of material contours $\left\{\boldsymbol{X}_{1}, \boldsymbol{X}_{2}, \ldots, \boldsymbol{X}_{n}\right\}$, across each of which $q$ jumps by a constant $\Delta q_{j}$, with the convention that the value of $q$ to the immediate left of the contour is $\Delta q_{j}$ plus the value of $q$ to the immediate right of the contour. CD provides equations of motion for each point $\boldsymbol{X}_{j}$ (represented by any arbitrary parametrisation) on a contour, namely

$$
\frac{\mathrm{d} \boldsymbol{X}_{j}}{\mathrm{~d} t}=\boldsymbol{u}\left(\boldsymbol{X}_{j}\right)
$$

or just advection of $\boldsymbol{X}_{j}$ by the velocity field $\boldsymbol{u}$. The latter depends on the instantaneous positions of all of the contours and the jumps $\Delta q_{j}$ across them, and may also include imposed flows such as a uniform rotation or translation.

For the QGSW model and with no imposed flow, the velocity at an arbitrary point $\boldsymbol{x}$ may be evaluated from

$$
\boldsymbol{u}(\boldsymbol{x})=\frac{1}{2 \pi} \sum_{k=1}^{n} \Delta q_{k} \oint_{C_{k}} K_{0}\left(\gamma\left\|\boldsymbol{x}-\boldsymbol{X}_{k}\right\|\right) \mathrm{d} \boldsymbol{X}_{k}
$$

where $K_{0}$ is the modified Bessel function of the second kind, and where $C_{k}$ denotes the $k$ th contour. Here, $-(2 \pi)^{-1} K_{0}(\gamma r)$ is the Green function for the operator $\nabla^{2}-\gamma^{2}$. When $\gamma \rightarrow 0$, this tends to $(2 \pi)^{-1} \ln r$, the Green function for Laplace's operator $\nabla^{2}$, plus an unimportant constant. When $\boldsymbol{x}=\boldsymbol{X}_{j}$, a point on one of the contours, Eqs.(7) and (8) fully determine the evolution of the flow. Points not on any contour contribute nothing to the dynamics. Note that while $K_{0}(\gamma r)$ is singular as $r \rightarrow 0$, the singularity is integrable and poses no difficulties numerically or, indeed, mathematically. The regularity of the CD equations was established by Chemin (1991) for the limit $\gamma \rightarrow 0$, and is expected to hold for arbitrary $\gamma$ since the singularity is no stronger.

Contour surgery (CS) is the numerical method used to solve Eqs.(7) and (8), taking each contour to be a finite set of 'nodes' connected by local cubic splines, and allowing topological changes, 'surgery', between two parts of the same contour, or between two different contours 


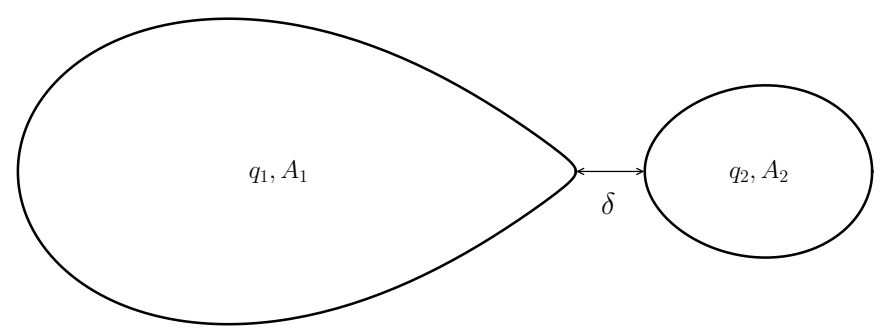

Figure 1. Schematic of the equilibrium configuration for two vortices having generally different areas $A_{1}$ and $A_{2}$ and different uniform values of $\mathrm{PV} q_{1}$ and $q_{2}$. The gap between the innermost edges of the vortices is $\delta$. This is decreased to sweep out a whole family of equilibria keeping all other parameters fixed.

separating the same PV values (Dritschel 1988, 1989a). Full details of the numerical method can be found in these references. In the results presented in $\S 4$, we use the standard setting $\mu=0.2$ for the dimensionless node separation (Fontane and Dritschel 2009), a characteristic length $L=\pi / 40$ (for initially two vortices of combined area $\pi$ ), and a time step $\Delta t=0.02$ (for vortices containing PV equal to $4 \pi$ or up to a multiple of 2 larger). With these parameter settings, the scale of surgery $\ell_{s}=\mu^{2} L / 4 \approx 8 \times 10^{-4}$. These parameter choices ensure accurate short to medium-term integrations (see below and Dritschel (2005)), and represent a trade-off between accuracy and efficiency. A smaller scale of surgery would lead to greater accuracy but at a prohibitively high numerical cost. Some of the more complex simulations reported below took more than two months of computer time on an Intel i7-3970X single-processor workstation.

\section{Vortex patch equilibria and their linear stability}

\subsection{Numerical methods}

We employ the same iterative method developed by Dritschel (1995) and used in Płotka and Dritschel (2013) to find doubly-connected QGSW vortex patch equilibria, with the only difference being that we allow the PV in each patch to be different. This does not result in any significant changes to the method.

The geometry of the problem is depicted in figure 1. Without loss of generality, we take the combined area of the two vortices to be $A_{1}+A_{2}=\pi$ and the combined circulation to be $\Gamma_{1}+\Gamma_{2}=\pi$ where $\Gamma_{j}=q_{j} A_{j}$. These choices effectively non-dimensionalise both space and time. An equilibrium then depends on four parameters: the area ratio $\alpha=A_{2} / A_{1}$, the circulation ratio $\xi=\Gamma_{2} / \Gamma_{1}$, the inverse Rossby deformation length $\gamma$, and the gap $\delta$ between the inner edges of the vortices. Note the PV ratio is determined from $\rho=q_{2} / q_{1}=\xi / \alpha$.

Numerically, each vortex is represented by 200 nodes, whose spacing is adjusted dynamically so that the travel time of a fluid particle between successive nodes is the same everywhere. We start with a guess - two circular vortices - when the vortices are well separated $(\delta=2$ is sufficient). The rotation rate $\Omega$ of the equilibrium is approximated by that corresponding to two 'point' vortices, i.e. concentrating the circulations of the two vortices at two points. Then the boundary shapes and rotation rate are corrected iteratively until the maximum change in the radius of any point on either contour falls below a tolerance, here $4 \times 10^{-8}$. The method ensures that the areas of the vortices are preserved, and that the gap between the inner edges of the vortices remains $\delta$. Once this first solution is found, we decrease $\delta$ in increments of $10^{-3}$ and find successive solutions in the same way. The method converges until a near corner forms on one (or both) of the vortices, marking the end of the branch of solutions.

Properties of the equilibria such as the vortex centres $\overline{\boldsymbol{x}}_{j}(j=1,2)$ and the angular impulse $\mathcal{J}$ in Eq.(5) are calculated from their equivalent contour-integral forms. The centres are found 


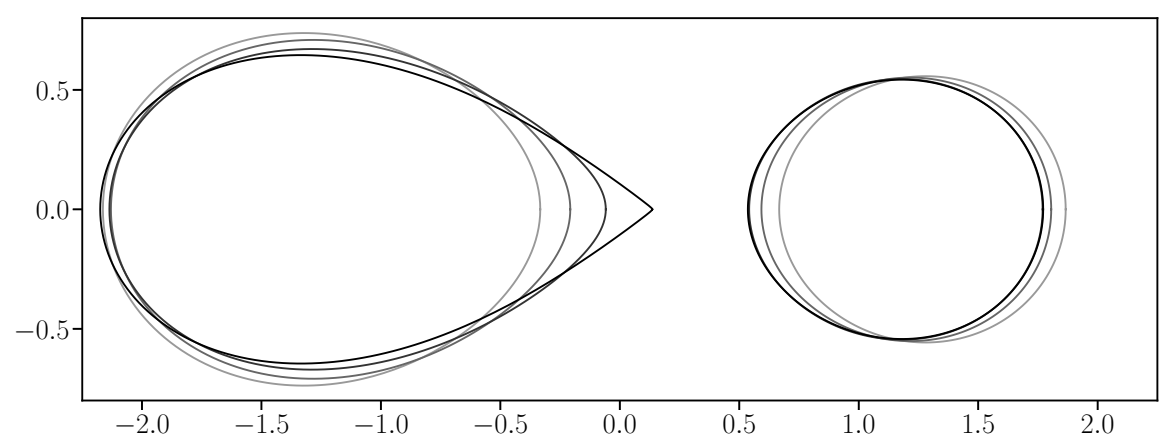

Figure 2. Equilibrium states two vortices having $A_{2} / A_{1}=0.5$ and $q_{2} / q_{1}=2$, for $\gamma=1$. Shown are 4 different values of the gap: $\delta=1$ (lightest grey), $\delta=0.8$ (light grey), $\delta=0.6$ (dark grey) and $\delta=0.4$ (black).

from

$$
\overline{\boldsymbol{x}}_{j}=\frac{1}{3 A_{j}} \oint_{C_{j}} \boldsymbol{X}_{j}\left(X_{j} \mathrm{~d} Y_{j}-Y_{j} \mathrm{~d} X_{j}\right)
$$

while the angular impulse is found from

$$
\mathcal{J}=\frac{1}{4} \sum_{j=1}^{2} \oint_{C_{j}}\left\|\boldsymbol{X}_{j}\right\|^{2}\left(X_{j} \mathrm{~d} Y_{j}-Y_{j} \mathrm{~d} X_{j}\right) .
$$

Numerically, 2-point Gaussian quadrature is used along the curved portions of the contours between successive nodes for high accuracy. The energy $\mathcal{E}$ can also be calculated from contour integrals (see Appendix B in Płotka and Dritschel 2012), but it provides no additional useful information not already contained in $\mathcal{J}$. Both $\mathcal{J}$ and $\mathcal{E}$ exhibit an extremum at the margin of stability, and thus only $\mathcal{J}$ is needed to locate the critical gap $\delta_{c}$ below which the equilibria are unstable (Płotka and Dritschel 2013).

The linear stability of each equilibrium is also calculated directly, both to verify that the minimum of $\mathcal{J}$ always coincides with the margin of stability and to explore the nonlinear consequences of various modes of instability existing for $\delta<\delta_{c}$ (see $\S 4$ below). The linear stability method is identical to that described in Appendix B of Dritschel (1995), apart from the Green function used; instead of $(2 \pi)^{-1} \ln r$, we use $-(2 \pi)^{-1} K_{0}(\gamma r)$. The same method was used by Płotka and Dritschel (2013) in a closely related study, including the number of modes representing boundary disturbances (100). The weak sensitivity of the method to this choice and to the number of nodes representing each vortex is discussed in Płotka and Dritschel (2013) and in the next subsection.

\subsection{An illustrative example}

We begin by examining a specific family of solutions for $\alpha=A_{2} / A_{1}=0.5, \rho=q_{2} / q_{1}=2$ and $\gamma=1$. This family is representative of all families studied. The vortex shapes are shown in figure 2 for 4 equally-spaced gaps, $\delta=1,0.8,0.6$ and 0.4 . The vortices become increasingly deformed from a circular shape as the gap decreases, and the last member of the family shown (darkest lines) exhibits a (near) corner on the innermost edge of vortex 1 (the corner state occurs for $\delta \approx 0.39$; this state cannot be accessed numerically from the method used). The smaller but more intense vortex 2 shows the weakest deformation, as expected since its larger PV protects it from the strain induced by vortex 1 (see $\S 4.1$ in Dritschel and de la Torre Juárez 1996). 

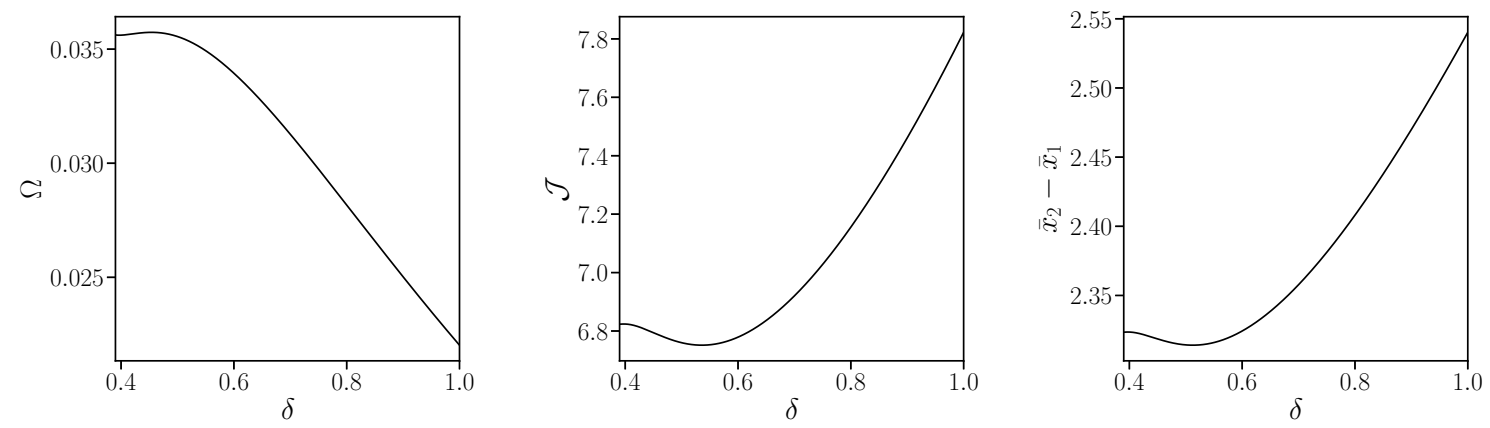

Figure 3. Properties of the equilibrium solutions plotted as a function of the gap $\delta$, for two vortices having $A_{2} / A_{1}=0.5$ and $q_{2} / q_{1}=2$, and for $\gamma=1$. The left panel shows the rotation rate $\Omega$, the middle panel shows the angular impulse $\mathcal{J}$, while the right panel shows the distance between the vortex centres $\bar{x}_{2}-\bar{x}_{1}$.

The limiting corner state occurs when the stagnation point normally located between the two vortices touches one of them. The contour generically turns through a right-angle at a corner (Overman and Zabusky 1982), where the local strain rate diverges like $\ln r$ where $r$ is the distance from the corner. Such corner states are not accessible via any evolution from a contour having a continuous tangent vector (Chemin 1991), and moreover are likely to have modes of instability with unbounded growth rates. For all families of solutions investigated, the margin of stability occurs before reaching the corner state, and growth rates are observed to rise sharply approaching the corner state (see below).

Various properties of the solutions as a function of the gap $\delta$ are shown in figure 3 . The rotation rate $\Omega$ generally increases with decreasing gap until the vortices become strongly deformed. Then $\Omega$ increases before again decreasing and so on, potentially doing this an infinite number of times before the theoretical corner state is reached (Luzzatto-Fegiz and Williamson 2010, 2011, Dritschel et al. 2018). A similar behaviour is exhibited by the angular impulse $\mathcal{J}$ and the centroid separation $\bar{x}_{2}-\bar{x}_{1}$, except these both decrease at first before rising and falling repeatedly. The form of these curves is similar for all area ratios, PV ratios and $\gamma$ investigated.

The linear stability analysis performed seeks shape deformations (eigenmodes) which evolve in time like $e^{-\mathrm{i} \sigma t}$, where $\sigma=\sigma_{r}+\mathrm{i} \sigma_{i}$ is the eigenvalue, the real part of which gives the frequency and the imaginary part of which gives the growth rate (or decay rate if negative). Note that, because the underlying dynamical system is Hamiltonian, if $\sigma$ is an eigenvalue, then so are $-\sigma, \sigma^{*}$ and $\sigma^{*}$, where $*$ denotes complex conjugation. In general, there are arbitrarily many eigenmodes but only a small number may be unstable (and all may be stable).

For the specific example considered in this subsection, figure 4 shows the growth rates and frequencies of a subset of the eigenmodes with decreasing gap (these have both $\left|\sigma_{r}\right|<3$ and $\left.\left|\sigma_{i}\right|<3\right)$. A pair of opposite-signed frequencies appear to coalesce, leading to the emergence of an unstable and a stable mode for $\delta<\delta_{c} \approx 0.53586$. The gap $\delta=\delta_{c}$ is where the angular impulse $\mathcal{J}$ reaches a minimum, and corresponds closely with the onset of linear instability. This is shown in detail in the zooms presented in figure 5 , showing how the margin of stability converges towards $\delta=\delta_{c}$ with increasing numerical resolution. The top panel shows the default resolution, in which each vortex is represented by 200 nodes, the error tolerance is $4 \times 10^{-8}$, and 100 modes are used to represent boundary disturbances in the linear stability analysis. The middle panel is for twice as many nodes and modes, and a quarter of the error tolerance. The bottom panel is for four times as many nodes and modes, and a sixteenth of the error tolerance. The results indicate that, with infinite resolution, the margin of stability would coincide with the location of the minimum in $\mathcal{J}$, as found previously in Dritschel (1995) and Płotka and Dritschel (2013). 


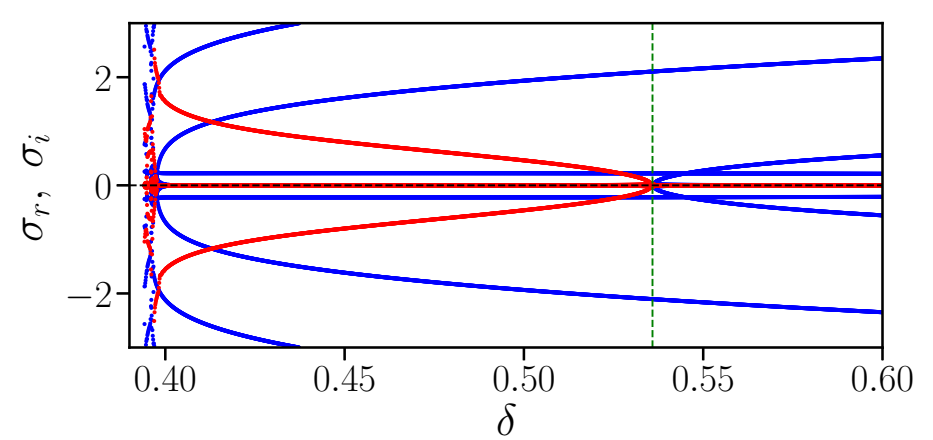

Figure 4. Frequencies $\sigma_{r}$ (blue) and growth rates $\sigma_{i}$ (red) as a function of the gap $\delta$, for two vortices having $A_{2} / A_{1}=0.5$ and $q_{2} / q_{1}=2$, and for $\gamma=1$. Note: there are many other higher frequency modes not visible in the domain of view. The green vertical dashed lines indicates the critical gap $\delta_{c}$ where the angular momentum $\mathcal{J}$ reaches a minimum.
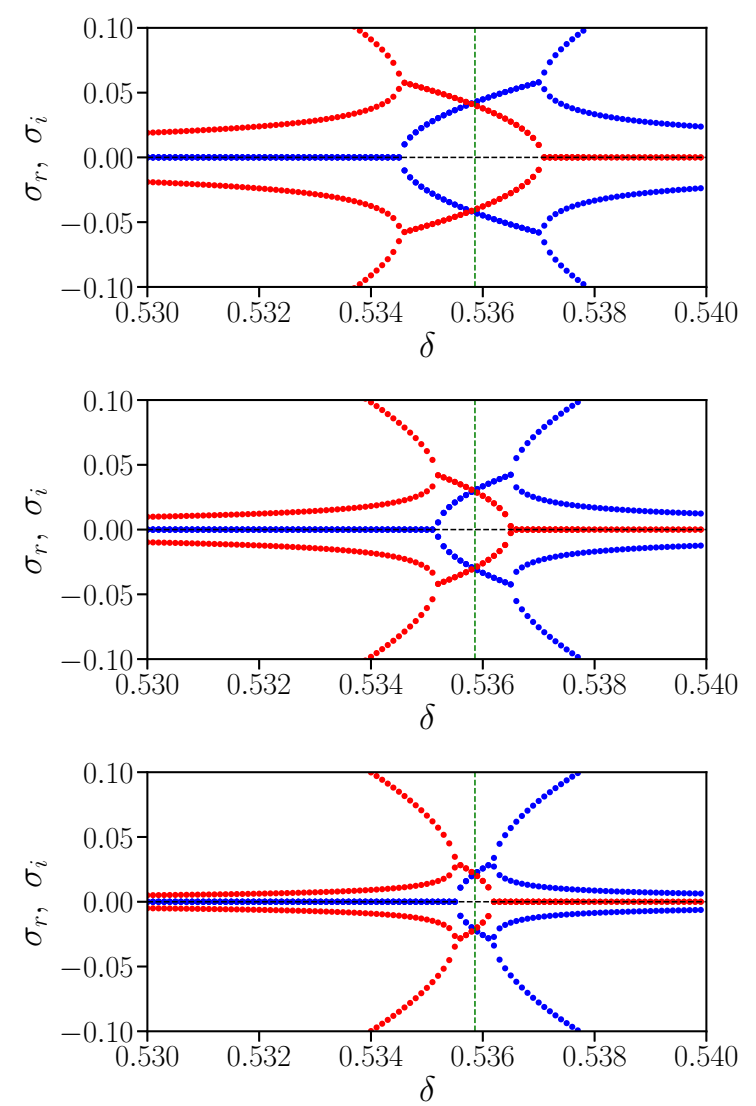

Figure 5. Zooms showing the frequencies $\sigma_{r}$ (blue) and growth rates $\sigma_{i}$ (red) near the critical gap $\delta_{c}$ (green vertical dashed line, for three different resolutions: default (top), double (middle) and quadruple (bottom).

For smaller gaps, the instabilities strengthen and new modes of instability appear just before the limiting corner state near $\delta=0.39$. The growth rate of the primary instability grows sharply near this gap. Near-corner states are generically highly unstable.

\subsection{Vortex properties at the margin of instability}

For like-signed vortices, the coincidence of the margin of stability with the first minimum in angular impulse $\mathcal{J}$ encountered with decreasing $\delta$ appears to occur for all $A_{2} / A_{1}, q_{2} / q_{1}$ and $\gamma$. 

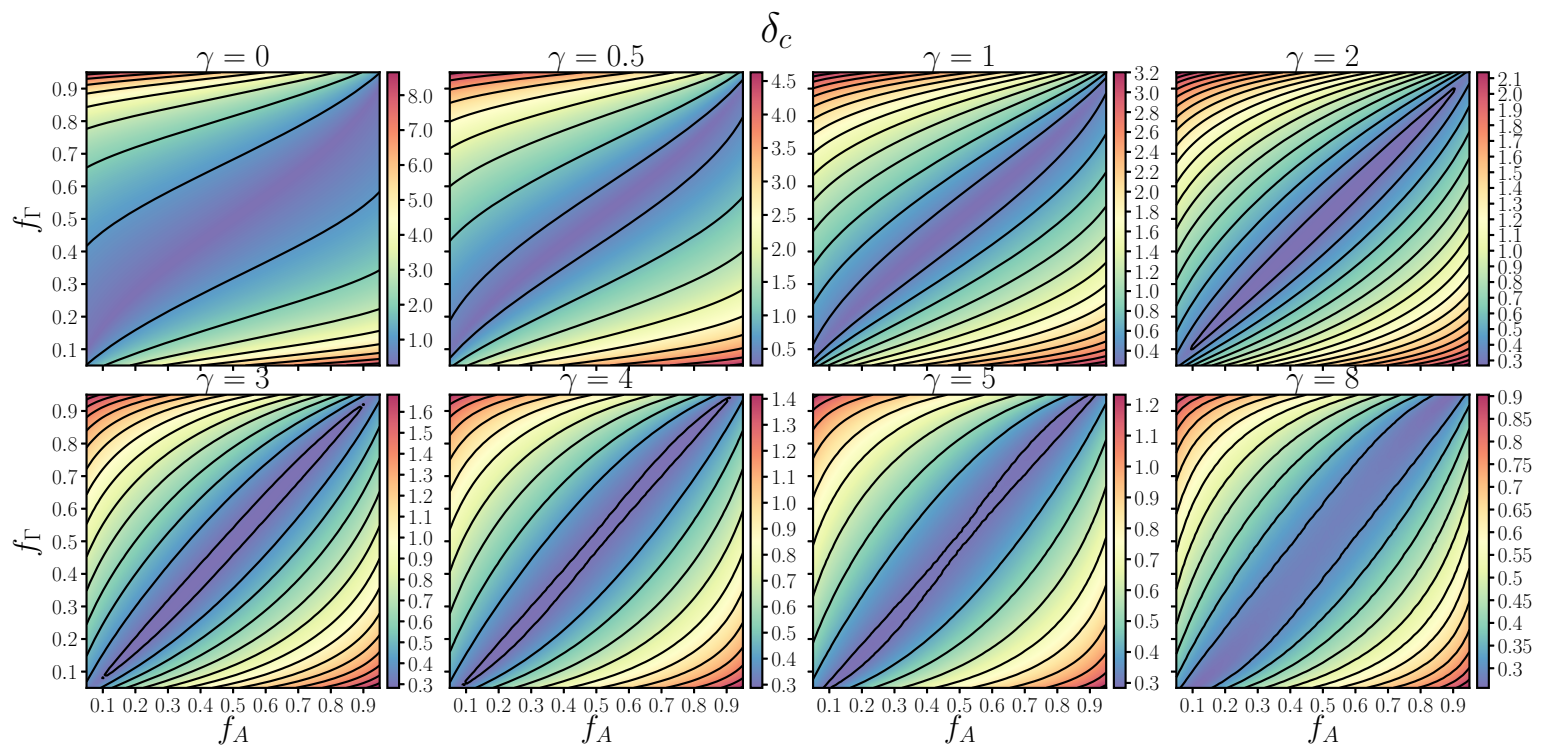

Figure 6. Critical gap $\delta=\delta_{c}$ below which equilibria are unstable, plotted as a function of the vortex area fraction $f_{A}=A_{2} /\left(A_{1}+A_{2}\right)$ and the vortex circulation fraction $f_{\Gamma}=\Gamma_{2} /\left(\Gamma_{1}+\Gamma_{2}\right)$, for several values of the inverse Rossby deformation length $\gamma$ as indicated. Note that the range in $\delta_{c}$ rapidly diminishes with $\gamma$.

This has been checked in tens of specific cases across parameter space. Hence, assuming this is true generally, we can determine the margin of stability over wide variations in parameters. Here, we fix $\gamma$ at a few illustrative values and vary the fractions $f_{A}=A_{2} /\left(A_{1}+A_{2}\right)$ and $f_{\Gamma}=\Gamma_{2} /\left(\Gamma_{1}+\Gamma_{2}\right)$ between 0 and 1 . This covers all possible area and PV ratios. From $f_{A}$ and $f_{\Gamma}$, we can recover the area, circulation and $\mathrm{PV}$ ratios from

$$
\begin{aligned}
\frac{A_{2}}{A_{1}} & =\alpha=\frac{f_{A}}{1-f_{A}} \\
\frac{\Gamma_{2}}{\Gamma_{1}} & =\xi=\frac{f_{\Gamma}}{1-f_{\Gamma}} \\
\frac{q_{2}}{q_{1}}=\rho & =\frac{\xi}{\alpha}=\frac{f_{\Gamma}\left(1-f_{A}\right)}{f_{A}\left(1-f_{\Gamma}\right)} .
\end{aligned}
$$

Note that the results are unchanged if we switch vortex 1 and vortex 2 . This means that results for a given $(\alpha, \xi)$ pair are identical to those for the $\left(\alpha^{-1}, \xi^{-1}\right)$ pair. In terms of the fractions $f_{A}$ and $f_{\Gamma}$, this means that the results for a given $\left(f_{A}, f_{\Gamma}\right)$ pair are identical to those for the $\left(1-f_{A}, 1-f_{\Gamma}\right)$ pair. Therefore we only have to generate results for $f_{\Gamma} \leq 1 / 2$, for all $f_{A}$, and use symmetry to obtain the results for $f_{\Gamma}>1 / 2$.

The critical gap $\delta=\delta_{c}$ where the first minimum in angular impulse $\mathcal{J}$ occurs when decreasing $\delta$ from $\infty$ is determined by interval division. First, three equilibrium states at equallyspaced gaps are found near the minimum in $\mathcal{J}$ by looking for a change in sign in the difference in $\mathcal{J}$ between successive states. Then the change in gap is halved and the process is repeated searching near the previous minimum. When the smallest change in $\mathcal{J}$ falls below $2.5 \times 10^{-7}$, the gap with the minimum $\mathcal{J}$ is considered to be the critical gap. This procedure is carried out for approximately $5000\left(f_{A}, f_{\Gamma}\right)$ pairs over half of the parameter plane for each $\gamma$ investigated. The other half is obtained by symmetry, as discussed above. For $\gamma=0$, a four times finer grid in $\left(f_{A}, f_{\Gamma}\right)$ is used since the numerical method is then significantly more efficient (avoiding the cost of evaluating modified Bessel functions).

The margin of stability or critical gap $\delta=\delta_{c}$ is shown in figure 6 for various values of $\gamma$, with the two-dimensional incompressible case $(\gamma=0)$ shown in the upper left panel. Equilibria 

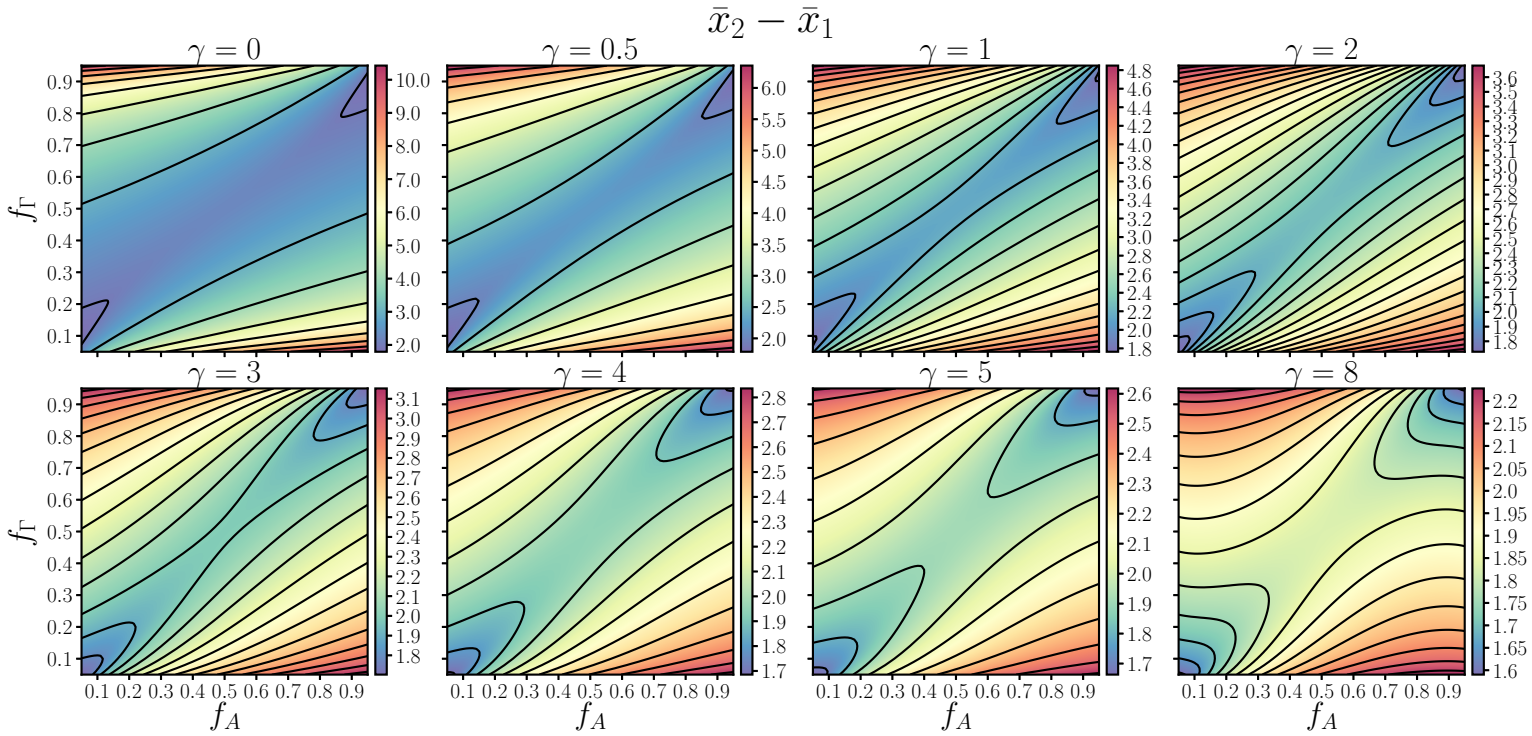

Figure 7. As in figure 6 but for the vortex centroid separation $\bar{x}_{2}-\bar{x}_{1}$ at the critical gap $\delta=\delta_{c}$. Note that the centroid separation rapidly diminishes with $\gamma$.

with gaps $\delta<\delta_{c}$ are unstable. (The fate of these instabilities is discussed in $\S 4$.) First of all, for each $\gamma$, the minimum critical gap occurs near the line $f_{\Gamma}=f_{A}$, which corresponds to $q_{2}=q_{1}$. Hence, vortices with nearly equal PV are stable over the greatest range of gaps. By contrast, vortices with the greatest disparity in PV, located in the upper left and lower right corners of the $\left(f_{A}, f_{\Gamma}\right)$ parameter plane, become unstable at the largest gaps. The dependence of $\delta_{c}$ is strongest on $f_{\Gamma}$ in these corners, but becomes more symmetric with $f_{A}$ as $\gamma$ increases. Moreover, for a fixed $\left(f_{A}, f_{\Gamma}\right)$ pair, $\delta_{c}$ rapidly decreases with $\gamma$, reflecting the shortening interaction range of the Green function, cf. Eq.(8). Vortices can be much closer together for $\gamma=8$ before they destabilise than for $\gamma=0$, particularly when there is a large disparity in PV.

The vortex centroid separation $\bar{x}_{2}-\bar{x}_{1}$ at the margin of stability is shown in figure 7 , using the same format as figure 6 . For small $\gamma$, the results resemble those for the critical gap $\delta_{c}$, with the minimum centroid separation occurring for nearly equal PV along $f_{\Gamma}=f_{A}$. For the special case of equal area and $\mathrm{PV}\left(f_{\Gamma}=f_{A}=0.5\right)$ and $\gamma=0$, the centroid separation at marginal stability is $\bar{x}_{2}-\bar{x}_{1}=2.2434$ or 3.1726 vortex radii (found using both 800 and 1600 nodes per vortex), which compares well to the estimate of 3.16 first obtained by Saffman and Szeto (1980). At slightly closer separations, Overman and Zabusky (1982) used CD simulations to verify that the equilibrium states are unstable, and that the two vortices eventually merge (further details including results for $\gamma>0$ may be found in Waugh (1992) and in $\S 4$ below).

As $\gamma$ increases, the smallest centroid separations are found in the lower left and upper right corners. Equilibria here also have similar values of PV but very different areas (and thus circulations). The lower left corner corresponds to a small area ratio, $\alpha=A_{2} / A_{1} \ll 1$, implying one vortex is much larger than the other (the same is true by symmetry in the upper right corner). The shortening interaction range with increasing $\gamma$ causes the vortices to become more circular and to approach each other more closely; two vortices essentially touch in the limit $\gamma \rightarrow \infty$ before becoming unstable. Hence, the smallest $\bar{x}_{2}-\bar{x}_{1}$ can get is $R_{1}+R_{2}$, the mean radius of each vortex. Since $A_{1}+A_{2}=\pi$, we find $R_{1}+R_{2} \rightarrow 1$ for $f_{A} \ll 1$ whereas $R_{1}+R_{2}=\sqrt{2}$ for $f_{A}=0.5$ (equal area). This is consistent with the observed variation of $\bar{x}_{2}-\bar{x}_{1}$ along $f_{\Gamma}=f_{A}$ seen for $\gamma=8$.

In the upper left and lower right corners, relatively large centroid separations are found. Here, one vortex is much larger but has much weaker circulation than the other, and hence 


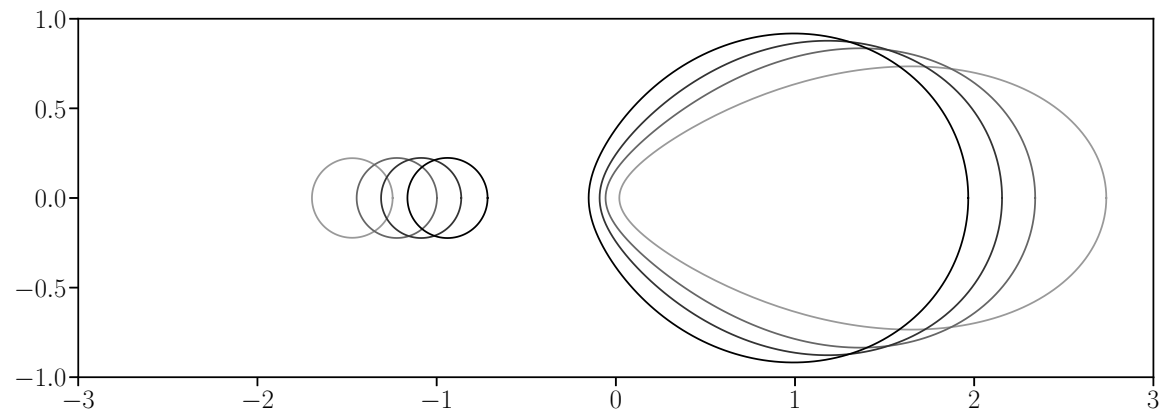

Figure 8. Equilibrium states at the margin of stability for two vortices having $f_{A}=0.95$ and $f_{\Gamma}=0.05$, for $\gamma=0$ (lightest grey), $\gamma=2$ (light grey), $\gamma=4$ (dark grey) and $\gamma=8$ (black). The corresponding gaps are $\delta=1.265,0.941$, 0.772 and 0.564 . Note, the area ratio $\alpha=19$ and the circulation ratio $\xi=1 / 19$, implying that the PV ratio $q_{2} / q_{1}=1 / 381$.
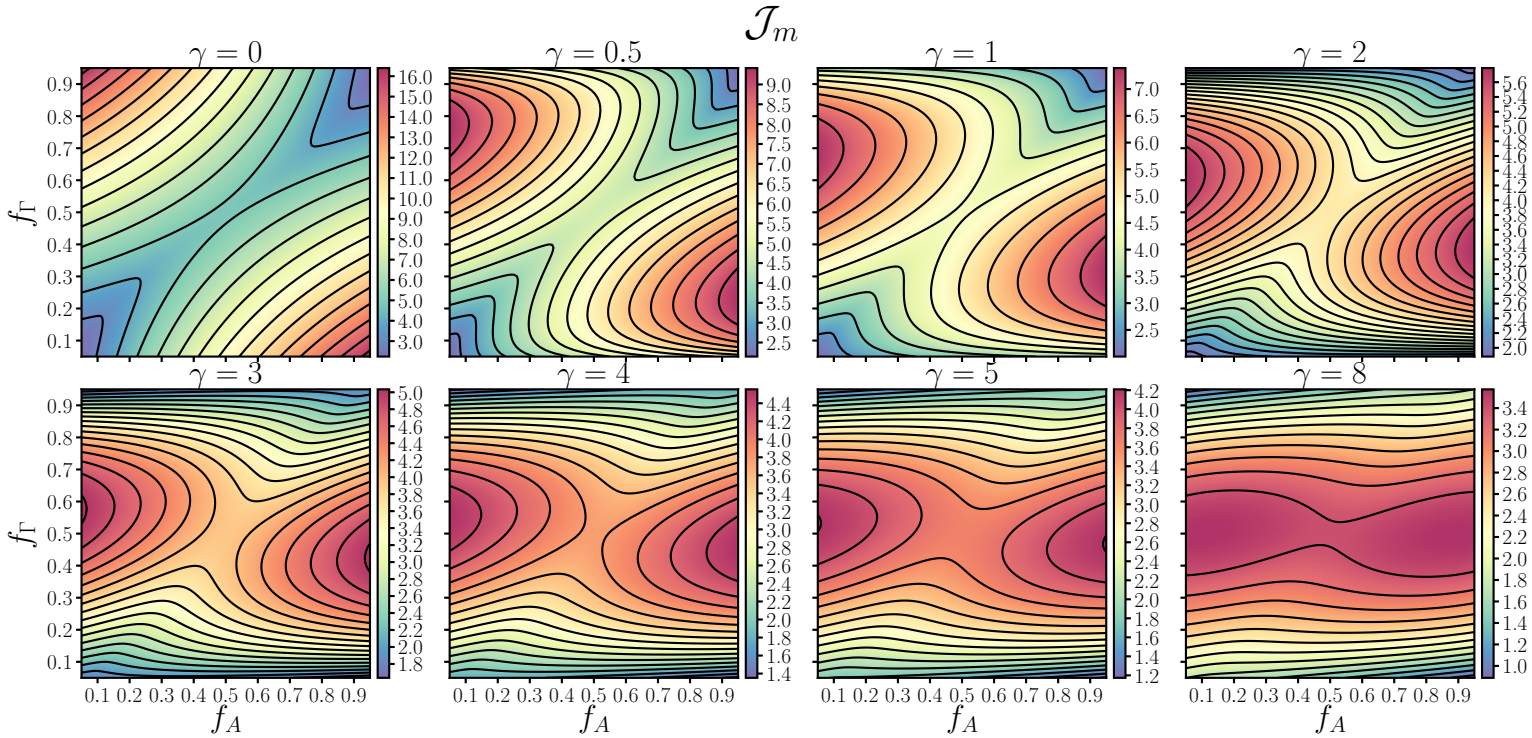

Figure 9. As in figure 6 but for the minimum angular impulse $\mathcal{J}_{m}$ at the critical gap $\delta=\delta_{c}$. Note that $\mathcal{J}_{m}$ rapidly diminishes with $\gamma$.

much weaker PV. In this case, the small vortex is nearly circular, behaving essentially like a point vortex, while the other vortex adopts a tear-drop shape to be in equilibrium. Examples are shown for equilibria at the margin of stability in figure 8 for $f_{A}=0.95$ and $f_{\Gamma}=0.05$, and for various $\gamma$. The most distortion occurs for $\gamma=0$ as expected. At large $\gamma$, the vortices can be much closer together.

We next consider the variation in the minimum angular impulse (versus $\delta$ ) with respect to $f_{A}, f_{\Gamma}$ and $\gamma$ in figure 9 . At small $\gamma$, the smallest values of $\mathcal{J}_{m}$ occur in the upper right and lower left corners, i.e. for nearly equal PV but strongly different area and circulation ratios. For these parameters, one vortex occupies a more central position while the other orbits around it. Comparable PV values enable both vortices to get relatively close without inducing strong deformation. For strongly different PV values, the vortex with the weaker PV is more readily distorted unless separated by a greater distance. This increases $\mathcal{J}_{m}$.

As $\gamma$ increases, the smallest values of $\mathcal{J}_{m}$ shift to the upper left and lower right corners, while the largest values are found centred on $f_{\Gamma}=0.5$, corresponding to vortices with equal circulation. In the upper left and lower right corners, the smaller but more intense vortex 

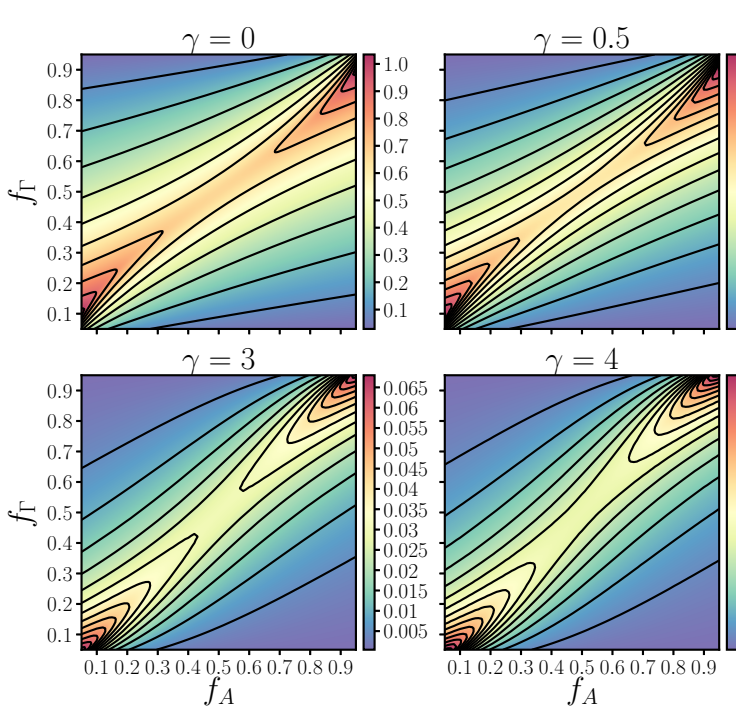

$\Omega$
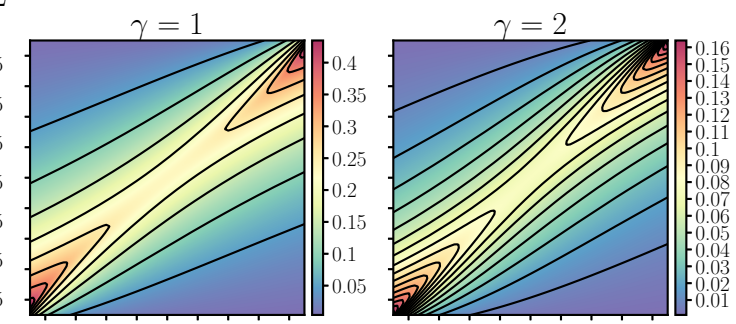

$\gamma=5$ $\gamma=8$
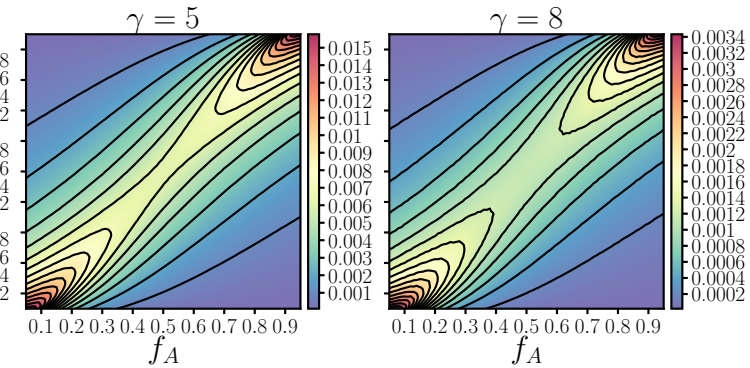

Figure 10. As in figure 6 but for the rotation rate $\Omega$ at the critical gap $\delta=\delta_{c}$. Note that $\Omega$, like the other diagnostics, rapidly diminishes with $\gamma$.

moves to the centre as seen in figure 8 , reducing $\mathcal{J}_{m}$, while the other vortex becomes less deformed, also reducing $\mathcal{J}_{m}$. The relatively high values of $\mathcal{J}_{m}$ occurring near $f_{\Gamma}=0.5$ when $\gamma=8$ are in fact lower than those occurring for smaller values of $\gamma$ in the same region of the $\left(f_{A}, f_{\Gamma}\right)$ parameter plane. Large $\gamma$ means the vortices can get closer before they become unstable, thereby reducing $\mathcal{J}_{m}$. Overall, $\mathcal{J}_{m}$ decreases as $\gamma$ increases.

Finally, we discuss how the equilibrium rotation rate $\Omega$ at marginal stability depends on parameters. This is shown in figure 10. For all $\gamma$, the largest rotation rates are found in the upper right and lower left corners, for nearly equal PV but for very different vortex areas and circulations. Here vortices tend to be closer (see figure 7), which is the main factor for the faster rotation. This also explains the curved shape of the ridge of relatively large $\Omega$. For small $\gamma$, this ridge curves above the line $f_{\Gamma}=f_{A}$ for small $f_{A}$, reaching it at $f_{A}=0.5$ then curves below it until $f_{A}=1$. Thus, a small vortex rotates faster around the larger vortex when the small vortex is a little more intense. The opposite occurs for large $\gamma$. Then, the small vortex rotates faster around the larger vortex when the small vortex is a little less intense. The small vortex needs just enough PV to withstand the shear exerted by the large vortex. For higher PV, the small vortex needs to orbit further out to avoid strongly distorting the large vortex.

With increasing $\gamma$, the rotation rates diminish rapidly as a consequence of the shortening range of the Green function. Maximum rotation rates are 300 times smaller for $\gamma=8$ than for $\gamma=0$. All time scales slow down, leading to ponderous motion. Płotka and Dritschel (2013) show that $\Omega$ scales like $\gamma^{-3}$ for $\gamma \gg 1$, whereas the frequency with which fluid particles orbit each boundary, $\Omega_{p}$, is much higher, scaling like $\gamma^{-1}$. Both are low frequencies, but the lower frequency controls the vortex interaction, both in equilibrium and out (see $\S 4$ ).

\section{Nonlinear dynamics}

We next consider the nonlinear evolution of weakly perturbed unstable equilibria near the margin of stability. The focus on marginally unstable states is motivated by the fact that such states are most relevant to vortex interactions occurring under the weak influence of other more distant vortices or external factors. As shown in Legras and Dritschel (1993) and Dritschel (1995), under these circumstances vortices evolve quasi-adiabatically, slowly evolving 
through a series of near equilibrium states. We envision therefore strong interactions occurring when the background flow pushes two vortices close enough together to become unstable. The marginally unstable equilibrium states are then likely to destabilise before they are pushed further together by the background flow.

As the parameter space is vast, we illustrate a few representative forms of vortex interactions based on approximately 100 simulations carried out. A comprehensive exploration of parameter space would be computationally prohibitive, and would be of limited utility for understanding vortex interactions in less idealised circumstances (Dritschel and Zabusky 1996). The effects of other more distant vortices, fronts, jets, topography, etc... all play a role in real geophysical flows, and should be taken into account in specific applications.

As found originally by Dritschel and Waugh (1992) for equal-PV vortex patch interactions in the two-dimensional incompressible model, and also by Yasuda and Flierl (1995) and Trieling et al. (2005) for unequal-PV, strong vortex interactions fall into four basic types, even when $\gamma>0$ (Płotka and Dritschel 2013). The first is complete merger (CM), where the two vortices form a single compound vortex encircled by thinning filamentary debris. This had previously been thought to be the only type of strong interaction, but in fact it occurs only for closely matched vortices (in area and in PV). The second type is partial merger (PM), in which one vortex looses material to the other but both survive. This is a much more common type of interaction than CM. The third type is complete straining out (CSO), in which one vortex is overwhelmed by the other and is stretched into an ever thinning filament. Finally, the fourth type is partial straining out (PSO), in which one vortex looses material to thinning filaments without being completely destroyed while the other vortex remains essentially unchanged.

Examples of the types of vortex interactions occurring are shown in figure 11. In the first column, the vortices initially have the same area but the vortex on the right has twice the PV. The more intense vortex tears a filament off the less intense one, reducing its area. This is an example of PSO.

In the second column, the vortex on the right has a quarter of the area of the one on the left but twice the PV. Despite the fact that the vortex on the right has less circulation, it pulls a large tongue of PV from the less intense vortex and becomes entrapped within it. Persistent holes are left in this weak ring of PV due to prior complex turbulent mixing. The initially larger vortex survives but at a much reduced size. This is an example of PM.

In the third column, the two vortices are equally matched in area and in PV, while $\gamma=0.02$ (close to the two-dimensional incompressible limit). The vortices initially merge with almost no loss of filamentary debris into a nearly perfectly elliptical vortex (see also Dritschel (1986)). The ellipse however is unstable and splits again just after $t=44$ into two vortices similar to the original equilibrium vortices, except very slightly asymmetric. The vortices then re-merge into a near ellipse and this destabilises asymmetrically, forming two vortices of significantly different area (see also supplementary movie 1). The intense boundary filamentation is typical for interactions with $\gamma \leq 1$ approximately. This is an example of CM followed by PM at late times.

In the final column, the two vortices are again equally matched in area and in PV but now $\gamma=4$, so long-range interactions are much weaker than in the third column. In this case, the two vortices merge into a barge-shaped vortex which nearly splits into two around $t=40$ before returning to a barge-shaped vortex. The evolution appears to then become periodic, oscillating between a dumbell-shaped and a barge-shaped vortex (see also supplementary movie 2). This is an example of CM, but with persistent strong oscillations. When $\gamma<2$, the interaction instead leads to PM (Płotka and Dritschel 2013).

The regularity of these oscillations is examined in figure 12. The vortex aspect ratio $\lambda$ and orientation (or phase) $\phi$ are obtained by fitting the entire PV distribution to a single elliptical patch having the same area, PV and second moments (the integrals of $x^{2}, x y$ and $y^{2}$ over the patch), see Melander et al. (1986). The power spectrum of $\lambda$ is obtained from the squared 


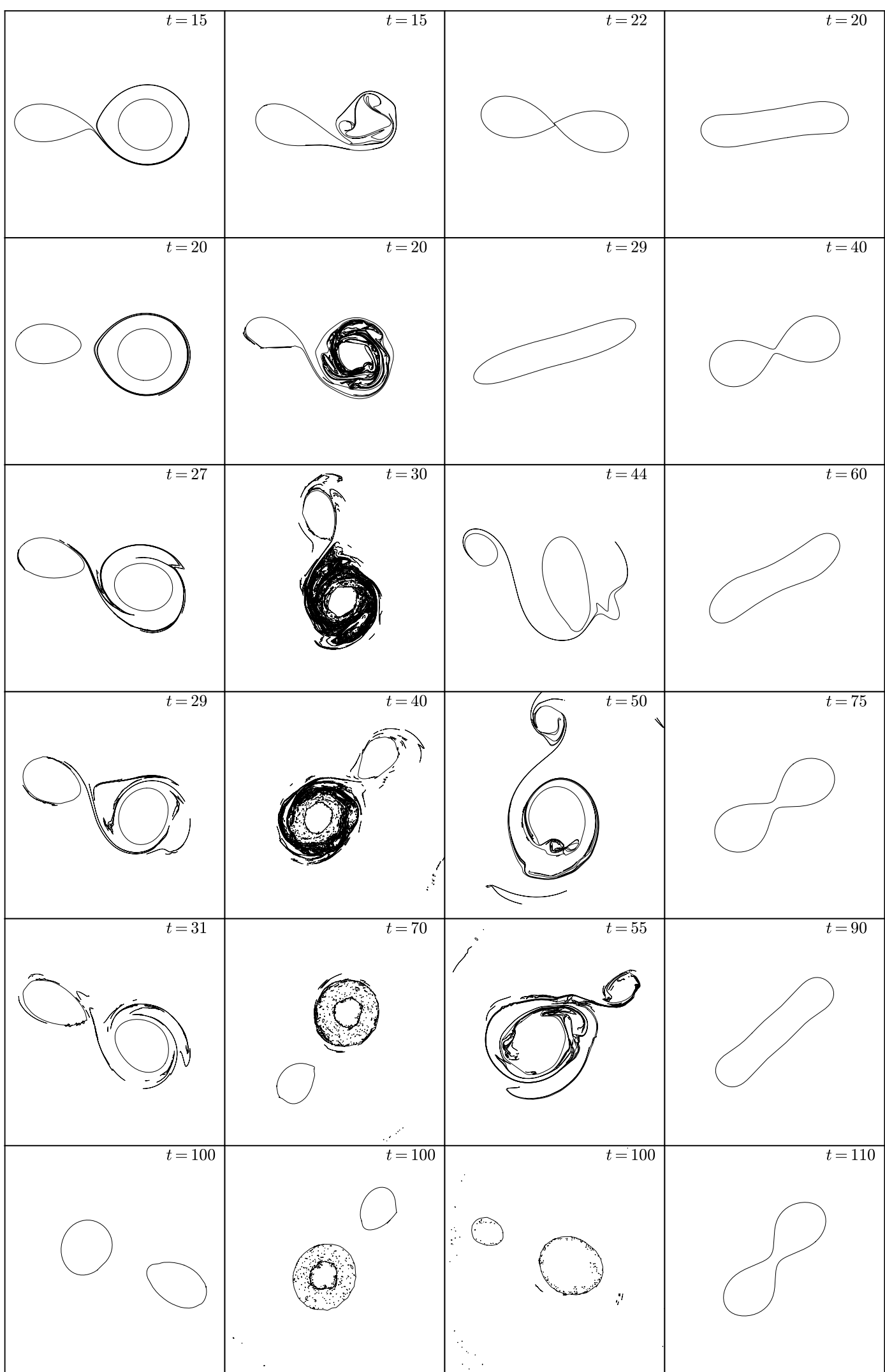

Figure 11. Four examples (left to right) of vortex interactions, with time increasing downwards as indicated. All examples start with marginally unstable equilibrium states perturbed only by weak numerical discretisation errors. In the 1st column, the area ratio $\alpha=A_{2} / A_{1}=1$, the PV ratio $\rho=q_{2} / q_{1}=2, \gamma=0.5$ and $\delta=0.69\left(\delta_{c}=0.79\right)$. In the 2nd column, $\alpha=0.25, \rho=2, \gamma=1$ and $\delta=0.265\left(\delta_{c}=0.435\right)$. In the 3rd column, $\alpha=1, \rho=1, \gamma=0.02$ and $\delta=0.129\left(\delta_{c}=0.259\right)$. In the 4th column, $\alpha=1, \rho=1, \gamma=4$ and $\delta=0.005\left(\delta_{c}=0.28\right)$. The window of view is $|x|<3$ and $|y|<3$. 

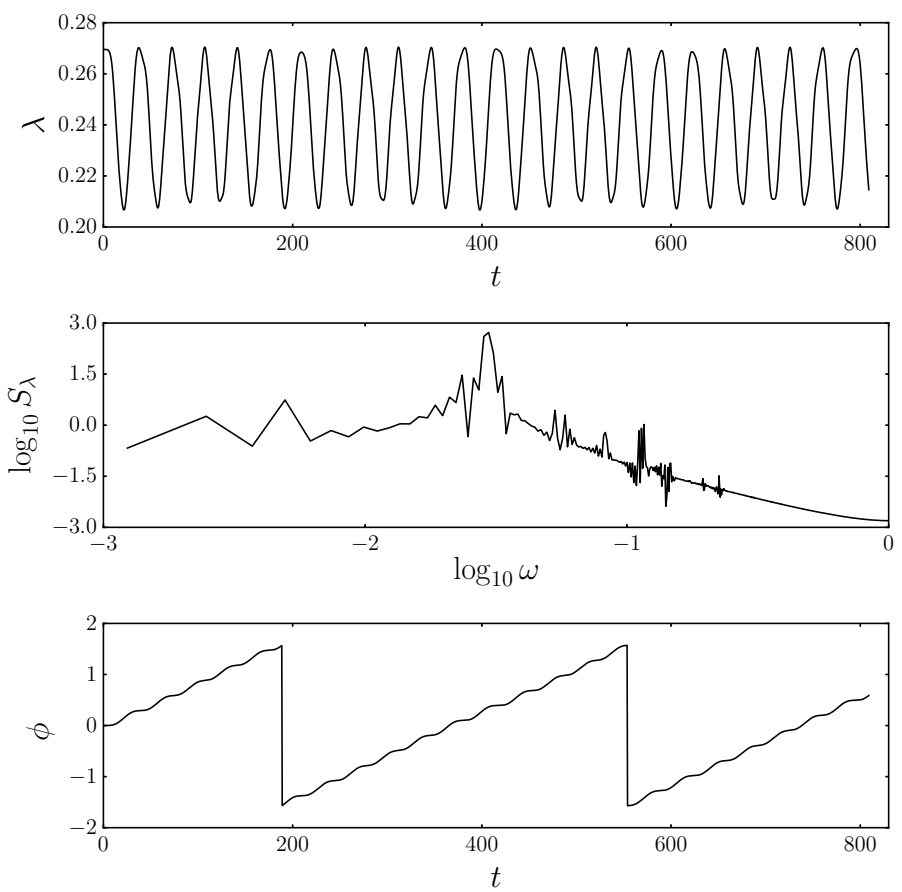

Figure 12. Aspect ratio $\lambda(t)$, associated frequency power spectrum $S_{\lambda}(\omega)$ and phase $\phi(t)$ of the moment fit ellipse corresponding to the vortex evolution shown in the 4 th column of figure 11.

magnitude of the Fourier coefficients for each frequency $\omega$ (in units of cycles per period). First of all, the aspect ratio shows quasi-regular oscillations, confirmed in the power spectrum by the strong peak around $\omega=0.029$ corresponding to a period of approximately 34.6 as observed in the time evolution of $\lambda$. The other, weaker peak at low frequency occurring around $\omega=0.0049$ corresponds to a period of approximately 203, again consistent with the broadening of the peaks and troughs in $\lambda(t)$ at multiples of this period. The phase $\phi(t)$ advances nearly linearly in time (it is constrained to lie between $-\pi / 2$ and $\pi / 2$ ). The small oscillations occur on the shorter period of 34.6 while the longer period is more difficult to distinguish. The entire vortex rotates only once in about 738 units of time due to the relatively large value of $\gamma$ (here 4).

We next consider the effect of $\gamma$ on vortex interactions of a given type. Figure 13 shows four examples of PM or PSO, with $\gamma$ increasing from 0.5 to 4 in factors of two from left to right. In all cases, the larger vortex is reduced in size and most of the material lost is tightly wrapped around the smaller but more intense vortex. Less material is lost as $\gamma$ increases, and less of it is wrapped around the other vortex, so that for $\gamma=4$ the interaction is more consistent with PSO rather than PM. However, the boundaries of both vortices are strongly agitated, containing waves at many scales, while the interior of the more intense vortex contains pockets of both irrotational fluid and fragments of the less intense vortex. (Supplementary movies 3 and 4 contrast the PM interaction in column 2 with the PSO one in column 4 , and better illustrate the evolution than still frames.) In all cases, the initially smaller vortex has less circulation but nonetheless dominates the interaction because of its larger PV. In fact, in no case examined with $\rho \neq 1$ does the more intense vortex get strained out by the less intense vortex, irrespective of the circulation ratio. The PV is the main factor controlling vortex survival.

Finally, figure 14 shows four examples of PSO, with $\gamma$ increasing from 0.5 to 4 in factors of two from left to right. These examples differ from those in figure 13 only in their initial area ratio: here $\alpha=1$ whereas previously $\alpha=0.25$. The increased size of the less intense vortex weakens the interaction, resulting only in a small to moderate loss of material from the less 


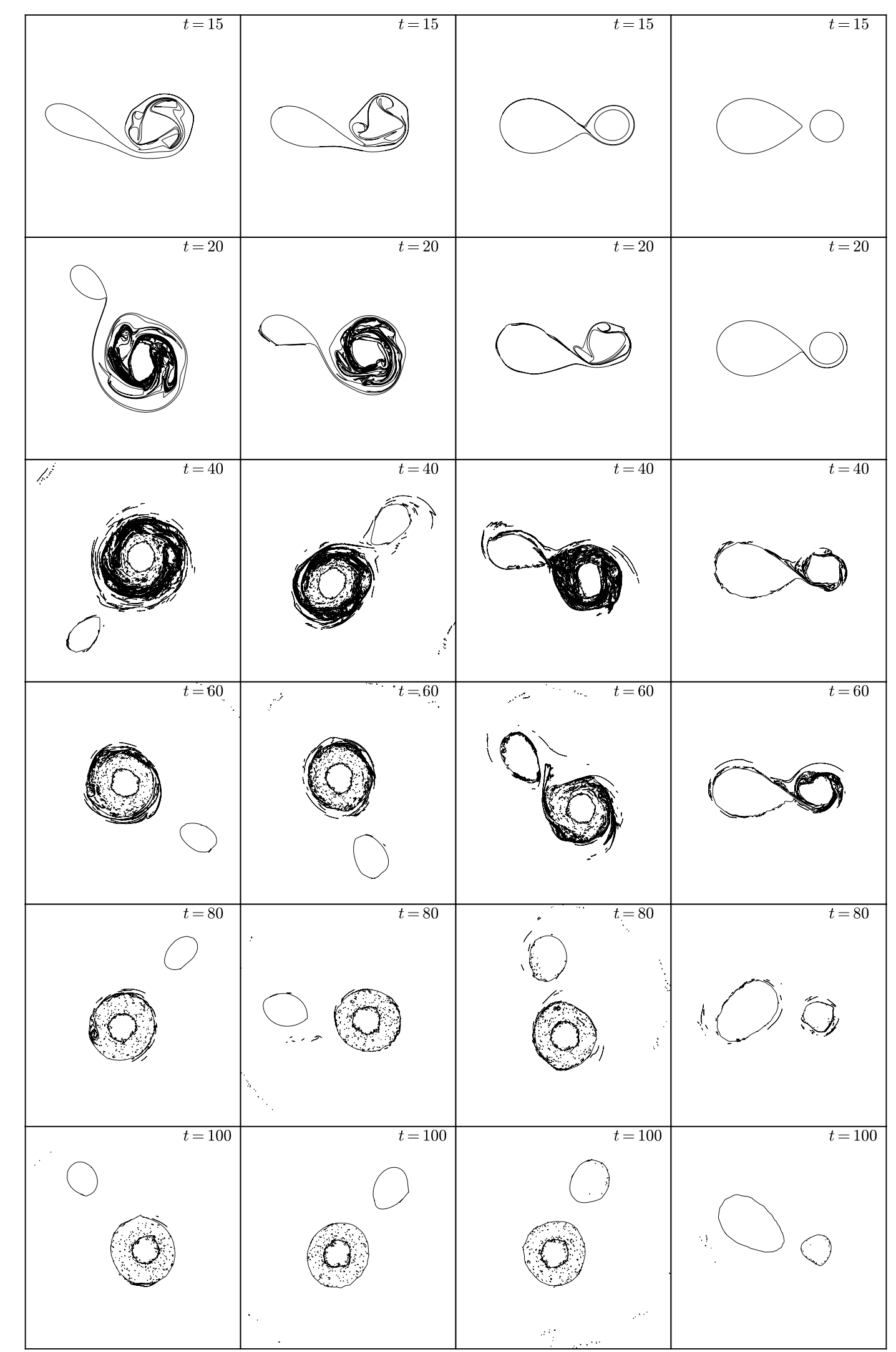

Figure 13. Four examples (left to right) of vortex interactions resulting in partial merger (PM) or partial straining out (PSO). Time increases downwards as indicated. All examples start with equilibria having $\alpha=0.25$ and $\rho=2$. Only $\gamma$ and $\delta$ differ. In the 1st column, $\gamma=0.5$ and $\delta=0.265\left(\delta_{c}=0.429\right)$. In the 2nd column, $\gamma=1$ and $\delta=0.265\left(\delta_{c}=0.435\right)$. In the 3rd column, $\gamma=2$ and $\delta=0.255\left(\delta_{c}=0.439\right)$. In the 4th column, $\gamma=4$ and $\delta=0.230\left(\delta_{c}=0.397\right)$. 


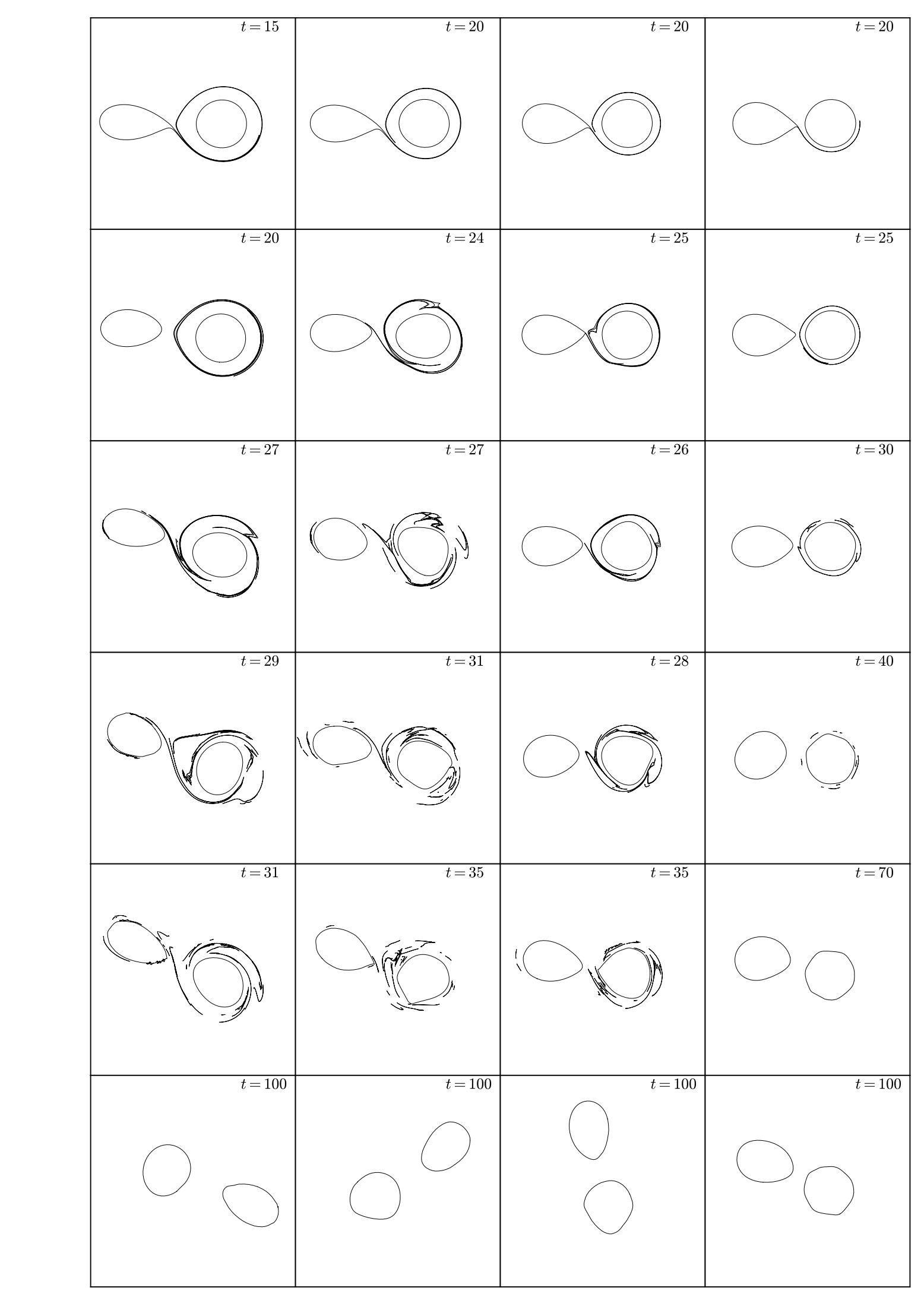

Figure 14. Four examples (left to right) of vortex interactions resulting in partial straining out (PSO). Time increases downwards as indicated. All examples start with equilibria having $\alpha=1$ and $\rho=2$. Only $\gamma$ and $\delta$ differ. In the 1 st column, $\gamma=0.5$ and $\delta=0.690\left(\delta_{c}=0.790\right)$. In the 2nd column, $\gamma=1$ and $\delta=0.600\left(\delta_{c}=0.650\right)$. In the 3rd column, $\gamma=2$ and $\delta=0.418\left(\delta_{c}=0.494\right)$. In the 4 th column, $\gamma=4$ and $\delta=0.262\left(\delta_{c}=0.383\right)$.
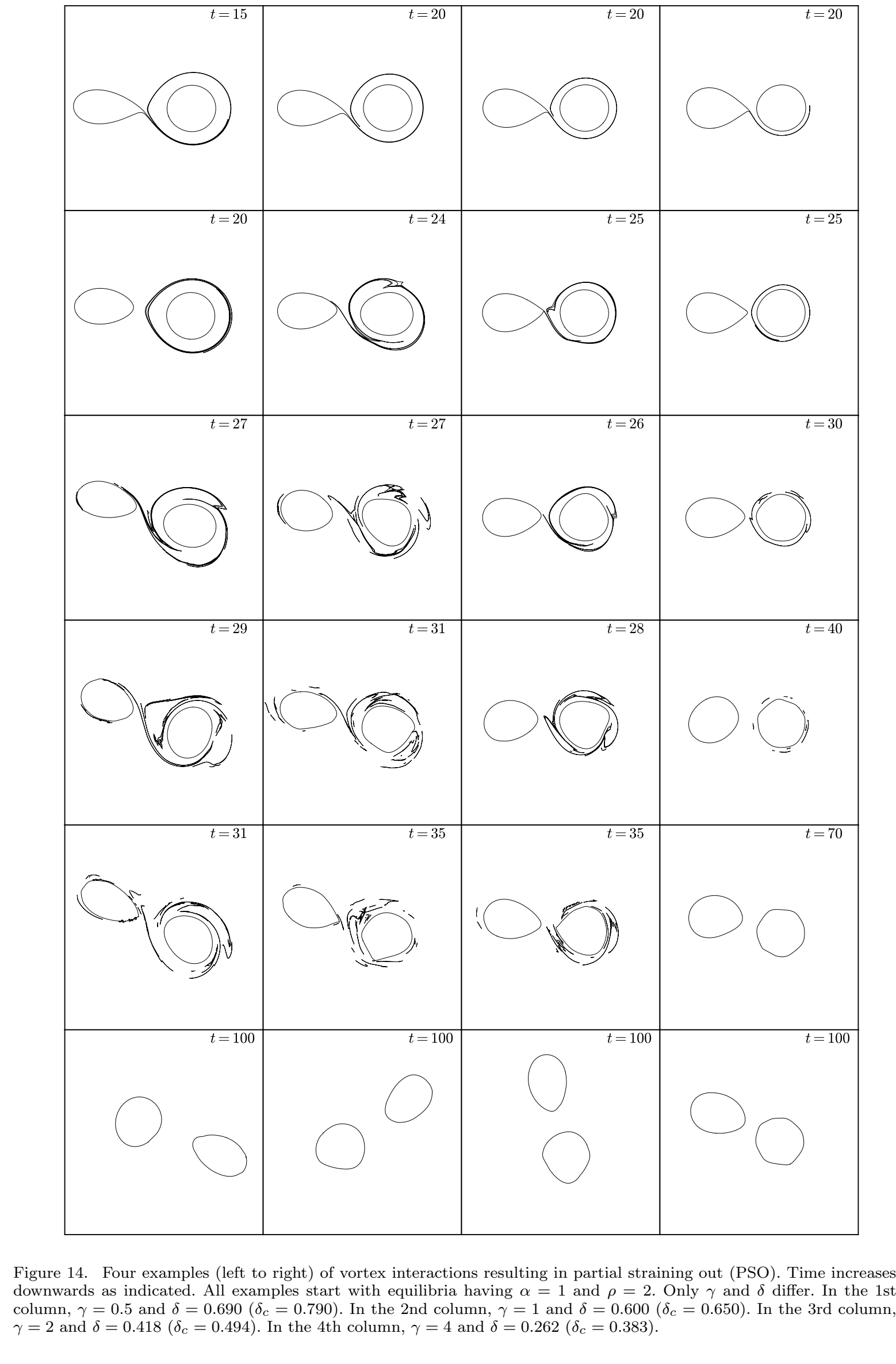


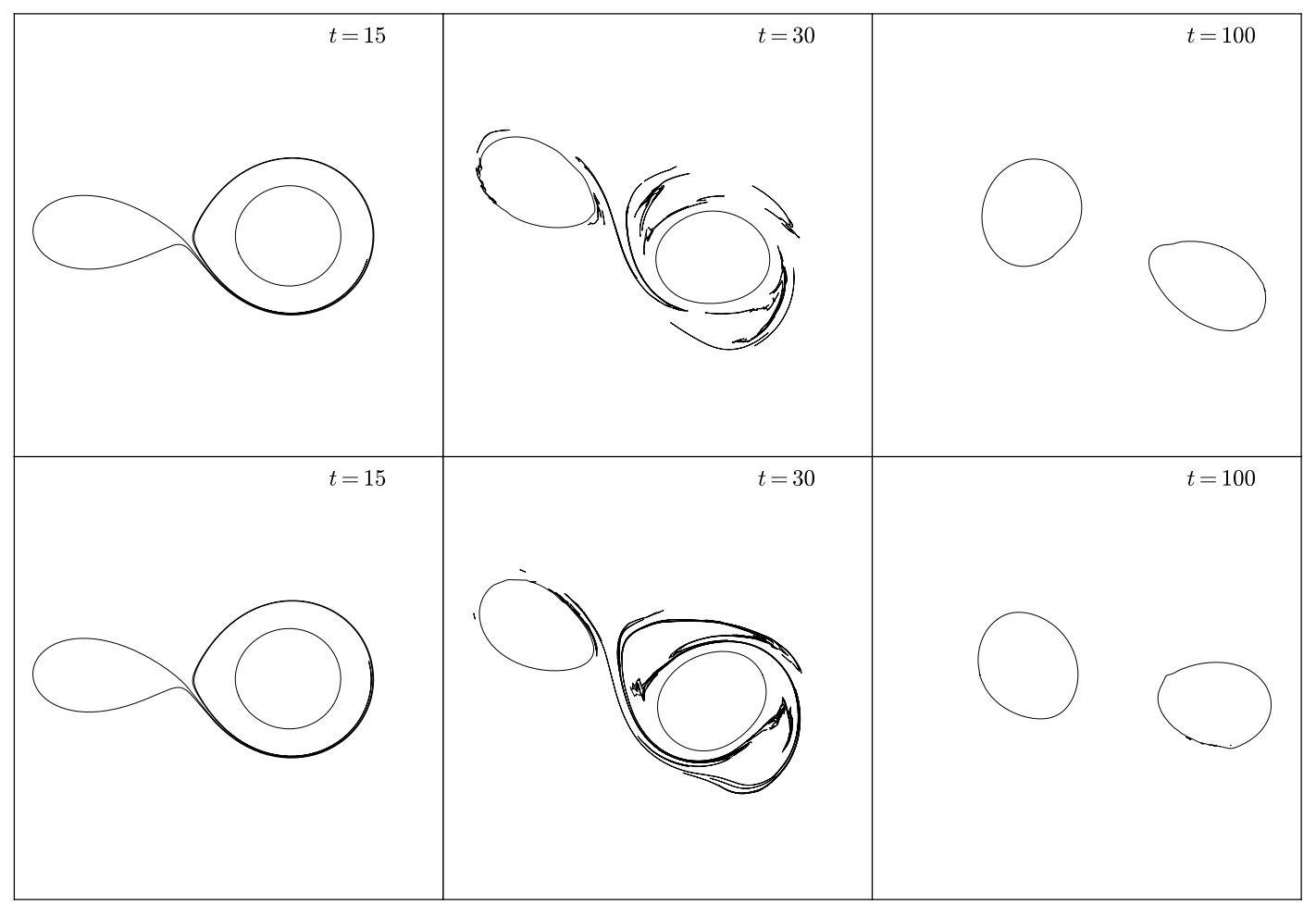

Figure 15. Comparison of two simulations with the scale of surgery differing by a factor of two, with the smaller scale of surgery shown in the bottom row. The example shown corresponds to the case shown in the left column in figure 14 , for $\alpha=1, \rho=2, \gamma=0.5$ and $\delta=0.69$. See text for further details.

intense vortex. This has little impact on the more intense vortex apart from exciting boundary waves and very small-scale filamentation. As $\gamma$ increases, the interaction weakens further and less material is torn away from the less intense vortex. In all cases, the weak filaments produced early on thin and are eventually eliminated by contour surgery at exceedingly small scales.

Over the long periods of integration reported, numerical errors associated with contour surgery and the finite representation of the contour shapes accrue, leading primarily to small changes in the positions and shapes of the vortices. This is examined in one case, corresponding to the left column in figure 14, by comparing with an identical simulation using half the scale of surgery (and half the characteristic length $L$, see $\S 2.2$ ). Results are shown in figure 15 , with the original simulation in the top row at times $t=15,30$ and 100, and with the higher-resolution simulation shown in the bottom row at the same times. The latter simulation was started at $t=8$, the time when the instability just becomes visible, by adding new nodes midway between the existing nodes in the original simulation. This is necessary because the instability grows only from numerical noise and would be delayed in the higher-resolution simulation if started at $t=0$. As previously demonstrated in Dritschel (2005), the early-intermediate evolution is only weakly affected by the scale of surgery. Here, many details match at $t=15$, apart from the lengths of the filaments as expected. By $t=30$ (corresponding to 30 rotations of the stronger vortex), differences begin to appear due to the effect of surgery. The removal of circulation around the more intense vortex results in a phase shift. Eventually, the errors amplify resulting in changes to both the shapes and locations of the vortices (see $t=100$ ), but the differences are not large. Qualitatively, the outcome of the instability is the same. Quantitatively, by $t=100$ the area of the less intense vortex differs by only $0.8 \%$, while the area of the more intense vortex differs by just $0.1 \%$. 


\section{Conclusions}

We have provided the first comprehensive description of the properties of doubly-connected vortex patch equilibria in the quasi-geostrophic shallow-water model. Such equilibria depend on 4 parameters: the vortex area ratio $\alpha$, the potential vorticity (PV) ratio $\rho$, the gap between their innermost edges $\delta$, and the inverse Rossby radius of deformation $\gamma$. The only restriction we have imposed is mirror symmetry across the line connecting the vortex centres. Other equilibria may exist without this symmetry, but they are not likely to be stable as they often bifurcate from symmetric solutions at an exchange in stability (Luzzatto-Fegiz and Williamson 2010, 2011, Dritschel et al. 2018).

We have found that the margin of (linear) stability generally coincides with the first minimum in the angular impulse $\mathcal{J}$ when decreasing $\delta$ from $\infty$. This allows one to efficiently map out the conditions at marginal stability, not only the critical gap $\delta_{c}$ within which all equilibria are linearly unstable, but also the vortex centroid separation $\bar{x}_{2}-\bar{x}_{1}$, minimum angular impulse $\mathcal{J}_{m}$ and rotation rate $\Omega$. We find that vortices with comparable values of PV can be closer together at marginal stability than vortices with disparate values. This indicates that the PV is the primary factor which protects a vortex from the strain exerted by another vortex, a result previously found in the two-dimensional incompressible model, i.e. $\gamma=0$ (Dritschel 1989b, Legras and Dritschel 1993, Mariotti et al. 1994, Yao et al. 1995, Dritschel 1995).

For symmetric vortices $(\alpha=\rho=1)$, perturbed equilibrium states just inside the margin of stability typically lead to vortex merger when $\gamma$ is not large (i.e. when $\gamma$ times the vortex radius is $O(1)$ or less) — see Overman and Zabusky (1982), Waugh (1992), Yasuda and Flierl (1995), Trieling et al. (2005), Brandt and Nomura (2010), Płotka and Dritschel (2013). But symmetry is very special. Slight departures from symmetry typically result in very different outcomes, most commonly partial merger where one vortex grows while the other shrinks in size (Dritschel and Waugh 1992, Dritschel 1995). Complete merger is uncommon.

In fact, weakly unstable equilibria very close to the margin of stability may not merge at all but instead exchange weak filaments and re-stabilise (Waugh 1992). We find the same situation in the present study: an equilibrium must be sufficiently unstable before symmetric merger, or any form of strong interaction occurs. As growth rates increase rapidly below the margin of stability (see figure 4), in practice the gap leading to a strong interaction is numerically close to the critical gap at the margin of stability ( $<10 \%$ of the mean vortex radius).

Asymmetric vortex patch interactions are highly varied, most often resulting in partial merger, partial straining out and complete straining out, as found in past studies (Dritschel and Waugh 1992, Yasuda and Flierl 1995, Trieling et al. 2005, Płotka and Dritschel 2013). Here we have additionally investigated the effect of the PV ratio for $\gamma>0$. When the PV ratio departs significantly from 1 (i.e. 2 or more), and the area ratio is less than 1 so that the smaller vortex is more intense, the most common form of interaction is partial merger. The larger, but less intense vortex becomes strongly deformed and expels a large tongue of PV that is wrapped around the smaller, more intense vortex. Meanwhile, part of the originally larger vortex recovers and moves further away where it is less affected by the other vortex. On the other hand, when one vortex has both larger PV and larger area, the typical interaction is partial or complete straining out of the smaller, less intense vortex.

The main effect of increasing $\gamma$ is to weaken all interactions, which favours the regimes of partial merger and partial straining out: as soon as one vortex begins to deform, conservation of angular impulse dictates that part of the vortex moves further away as the other part moves toward the other vortex. Due to the short interaction range for $\gamma \gg 1$, a small increase in the separation distance is enough to prevent the outer part of the deformed vortex from being strained out. The inner part may eventually end up around the periphery of the other vortex (partial merger) or be stretched into ever thinning filamentary debris (partial straining out). 
The results of this study, while idealised, provide a detailed and comprehensive picture of the stability and nonlinear evolution of pairs of like-signed vortices across a very wide parameter space in the QGSW model. As such, the results can broadly be applied to better understand naturally-occurring vortices and their interactions in geophysical flows. Rarely are pairs of vortices isolated however, and other effects which we have neglected, such as the background planetary vorticity variation and ageostrophic motions, do play a role, sometimes an important one, in real flows. Nonetheless, this study lays the foundation for more detailed studies, and at the very least identifies fundamental forms of interactions and their parameter dependence.

In future research, an important next step is to examine opposite-signed vortex equilibria and their interactions for general $\alpha, \rho$ and $\gamma$ (see Polvani (1988), Polvani et al. (1989), Polvani (1991), Dritschel (1995) and Makarov et al. (2012) and references therein for various special cases). This presents a challenge for two reasons: (1) there is no single parameter like the gap which monotonically varies along a solution branch, and (2) the margin of stability does not coincide with a minimum in the angular impulse $\mathcal{J}$ (Dritschel 1995). The first problem can be overcome by using $\Omega$ and $\mathcal{J}$ as control variables, as in Dritschel et al. (2018), to trace the full solution branch up to a corner state (see also Luzzatto-Fegiz and Williamson (2010, 2011)). The second problem requires one to perform a full linear stability analysis for each equilibrium to locate the margin of stability, a much more costly exercise. We hope to report on these results in the near future.

Additionally, it is important to examine the role played by ageostrophic motions and inertiagravity waves, both neglected in the QGSW model but present to varying degrees in real geophysical flows. Likewise, we could greatly enrich our understanding of such flows by including broader environmental effects like a background planetary vorticity variation, sloping bottom topography, and horizontal shear induced by distant vortices or fronts. And then there are three-dimensional effects to consider. There has been progress in all of these areas but much remains to be done.

\section{References}

Aref, H., 150 Years of Vortex Dynamics. Theor. Comput. Fluid Dyn., 2010, 24, 1-7.

Beron-Vera, F., Wang, Y. and Olascoaga, M., Objective detection of oceanic eddies and the Agulhas leakage. J. Phys. Oceanogr., 2013, 43, 1426-1438.

Brandt, L. and Nomura, K., Characterization of the interactions of two unequal co-rotating vortices. J. Fluid Mech., 2010, 646, 233-253.

Burgess, B., Dritschel, D. and Scott, R., Extended scale invariance in the vortices of freely evolving twodimensional turbulence. Phys. Rev. Fluids, 2017, 2, 114702.

Carton, X., Hydrodynamical Modeling Of Oceanic Vortices. Surveys in Geophysics, 2001, 22, 179-263.

Carton, X., Oceanic Vortices. In Fronts, Waves and Vortices in Geophysical Flows, edited by J.B. Flor, Vol. 805, chap. 3, pp. 61-108, 2010 (Springer-Verlag: Berlin).

Chemin, J.Y., Existence globalle pour le problème des poches de tourbillon. CR Acad. Sci. Paris Ser. I, 1991, pp. 803-806.

Deem, G. and Zabusky, N., Stationary V-states, interactions, recurrence, and breaking. In Solitons in Action (eds. K. Longren and A. Scott), 1978a, pp. 277-293.

Deem, G. and Zabusky, N., Vortex waves: stationary V-states, interactions, recurrence, and breaking. Phys. Rev. Lett., 1978b, 40, 859-862.

Dritschel, D., The stability and energetics of corotating uniform vortices. J. Fluid Mech., 1985, 157, 95-134.

Dritschel, D., The nonlinear evolution of rotating configurations of uniform vorticity. J. Fluid Mech., 1986 , 172, 157-182.

Dritschel, D., Contour surgery: A topological reconnection scheme for extended integrations using contour dynamics. J. Comput. Phys., 1988, 77, 240-266.

Dritschel, D., Contour dynamics and contour surgery: Numerical algorithms for extended, high-resolution modelling of vortex dynamics in two-dimensional, inviscid, incompressible flows. Computer Physics Reports, 1989a, 10, 77-146.

Dritschel, D., Strain-induced vortex stripping. In Mathematical aspects of vortex dynamics, edited by R. Caflisch, pp. 107-119, 1989b (Society for Industrial and Applied Mathematicsb: Philadelphiab). 
Dritschel, D., A general theory for two-dimensional vortex interactions. J. Fluid Mech., 1995, 293, 269-303.

Dritschel, D., Contour Dynamics. In Encyclopedia of Nonlinear Science, edited by A. Scott, pp. 168-171, 2005 (Routledge, Taylor \& Francis Group: New York).

Dritschel, D. and de la Torre Juárez, M., The instability and breakdown of tall columnar vortices in a quasigeostrophic fluid. J. Fluid Mech., 1996, 328, 129-160.

Dritschel, D., Hmidi, T. and Renault, C., Imperfect bifurcation for the quasi-geostrophic shallow-water equations. Archive for Rational Mechanics and Analysis, 2018 In press.

Dritschel, D. and Saravanan, R., Three-dimensional quasi-geostrophic contour dynamics, with an application to stratospheric vortex dynamics. Quart. J. Roy. Meteorol. Soc., 1994, 120, 1267-1297.

Dritschel, D. and Waugh, D., Quantification of the inelastic interaction of unequal vortices in two-dimensional vortex dynamics. Phys. Fluids A, 1992, 4, 1737-1744.

Dritschel, D. and Zabusky, N., On the nature of vortex interactions and models in unforced nearly-inviscid two-dimensional turbulence. Phys. Fluids, 1996, 8, 1252-1256.

Dunkerton, T. and Delisi, D., Evolution of potential vorticity in the winter stratosphere of January-February 1979. J. Geophys. Res., 1986, 91(D1), 1199-1208.

Fontane, J. and Dritschel, D., The HyperCASL Algorithm: a new approach to the numerical simulation of geophysical flows. J. Comput. Phys., 2009, 228, 6411-6425.

Ford, R., McIntyre, M. and Norton, W., Balance and the slow quasimanifold: some explicit results. J. Atmos. Sci., 2000, 57, 1236-1254.

Hmidi, T. and Mateu, J., Bifurcation of rotating patches from Kirchhoff vortices. Discrete Contin. Dyn. Syst., 2016, 36, 5401-5422.

Hoskins, B., McIntyre, M. and Robertson, A., On the use and significance of isentropic potential-vorticity maps. Quart. J. Roy. Meteorol. Soc., 1985, 111, 877-946.

Ingersoll, A., Dowling, T., Gierasch, P., Orton, G., Read, P., Sanchez-Lavega, A., Showman, A., Simon-Miller, A. and Vasavada, A., Dynamics of Jupiter's atmosphere. In Jupiter: the Planet, Satellites, and Magnetosphere, edited by F. Bagenal, chap. 6, pp. 105-128, 2007 (Cambridge University Press: Cambridge).

Kamm, J., Shape and stability of two-dimensional uniform vorticity regions. Ph.D. Thesis, California Institute of Technology, Pasedena, California, USA, 1987.

Kirchhoff, G., Vorlesungenb̈er mathematische Physik. Mechanik, 1876 (Leipzig: Teubner).

Kizner, Z., Reznik, G., Fridman, B., Khvoles, R. and McWilliams, J., Shallow-water modons on the f-plane. J. Fluid Mech., 2008, 603, 305-329.

Lamb, H., Hydrodynamics, 1932 (New York: Dover).

Legras, B. and Dritschel, D., Vortex stripping and the generation of high vorticity gradients in two-dimensional flows. Applied Scientific Research, 1993, 51, 445-455.

Love, A., On the stability of certain vortex motions. Proc. Lond. Math. Soc., 1893, 35, 18-42.

Luzzatto-Fegiz, P. and Williamson, C., Stability of elliptical vortices from "Imperfect-Velocity-Impulse" diagrams. Theor. Comput. Fluid Dyn., 2010, 24, 181-188.

Luzzatto-Fegiz, P. and Williamson, C., An efficient and general numerical method to compute steady uniform vortices. J. Comput. Phys., 2011, 230, 6495-6511.

Makarov, V. and Kizner, Z., Stability and evolution of uniform-vorticity dipoles. J. Fluid Mech., 2011, 672, $307-325$.

Makarov, V., Sokolovskiy, M. and Kizner, Z., Doubly symmetric finite-core heton equilibria. J. Fluid Mech., 2012, 708, 397-417.

Mariotti, A., Legras, B. and Dritschel, D., Vortex stripping and the erosion of coherent structures in twodimensional flows. Phys. Fluids, 1994, 6, 3954-3962.

McIntyre, M., The stratospheric polar vortex and sub-vortex: fluid dynamics and midlatitude ozone loss. Phil. Trans. Roy. Soc., 1995, 352, 227-240.

McIntyre, M. and Palmer, T., Breaking planetary waves in the stratosphere. Nature, 1983, 305, 593-600.

McKiver, W. and Dritschel, D., Balance in non-hydrostatic rotating stratified turbulence. J. Fluid Mech., 2008, 596, 201-219.

Melander, M., Zabusky, N. and McWilliams, J., Asymmetric vortex merger in two-dimensions: Which vortex is victorious?. Phys. Fluids, 1987, 30, 2610-2612.

Melander, M., Zabusky, N. and Styczek, A., A moment model for vortex interactions of the two-dimensional Euler equations. 1. Computational validation of a Hamiltonian elliptic representation. J. Fluid Mech., 1986, 167, 95-115.

Moffatt, H., Vortex dynamics: The Legacy of Helmholtz and Kelvin. In IUTAM Symposium on Hamiltonian Dynamics, Vortex Structures, Turbulence. IUTAM Bookseries, edited by A. Borisov, V. Kozlov, I. Mamaev and M. Sokolovskiy, Vol. 6, pp. 1-10, 2008 (Springer: Dordrecht).

Mohebalhojeh, A. and Dritschel, D., Hierarchies of balance conditions for the $f$-plane shallow water equations. J. Atmos. Sci., 2001, 58, 2411-2426.

Mohebalhojeh, A. and Dritschel, D., Contour-advective semi-Lagrangian algorithms for many-layer primitive equation models. Quart. J. Roy. Meteorol. Soc., 2004, 130, 347-364.

Overman, E. and Zabusky, N., Evolution and merger of isolated vortex structures. Phys. Fluids, 1982, 25, $1297-1305$.

Pedlosky, J., Geophysical Fluid Dynamics, 1979 (New York: Springer-Verlag).

Phillips, N., Energy transformations and meridional circulations associated with simple baroclinic waves in a 
two-level, quasigeostrophic model. Tellus, 1954, 6, 273-286.

Pierrehumbert, R., A family of steady, translating vortex pairs with distributed vorticity. J. Fluid Mech., 1980, 99, 129-144.

Płotka, H. and Dritschel, D., Quasi-geostrophic shallow-water vortex-patch equilibria and their stability. Geophys. Astrophys. Fluid Dyn., 2012, 106, 574-595.

Płotka, H. and Dritschel, D., Quasi-geostrophic shallow-water doubly-connected vortex equilibria and their stability. J. Fluid Mech., 2013, 723, 40-68.

Polvani, L., Geostrophic Vortex Dynamics. Ph.D. Thesis, Massachusetts Institute of Technology, 77 Massachusetts Avenue, Cambridge, MA MIT/WHOI WHOI-88-48, 1988.

Polvani, L., Two-layer geostrophic vortex dynamics. Part 2. Alignment and two-layer V-states. J. Fluid Mech., 1991, 225, 241-270.

Polvani, L., Zabusky, N. and Flierl, G., Two-layer geostrophic vortex dynamics. Part 1. Upper-layer V-states and merger. J. Fluid Mech., 1989, 205, 215-242.

Read, P., Dynamics and circulation regimes of terrestrial planets. Planetary and Space Sci., 2011, 59, 900-914.

Robinson, A., Luyten, J. and Flierl, G., On the theory of thin rotating jets: A quasigeostrophic time dependent model. Geophysical Fluid Dynamics, 1975, 6, 211-244.

Saffman, P. and Szeto, R., Equilibrium shapes of a pair of equal uniform vortices. Phys. Fluids, 1980, 23, 2339-2342.

Samelson, R., Schlax, M. and Chelton, D., Randomness, Symmetry, and Scaling of Mesoscale Eddy Life Cycles. J. Phys. Oceanogr., 2014, 44, 1012-1029.

Thomson, W.L.K., On vortex motion. Trans. Roy. Soc. Edin., 1869, 25, 217-260.

Trieling, R., Fuentes, O. and van Heijst, G., Interaction of two unequal corotating vortices. Phys. Fluids, 2005, 17, 087103.

Vallis, G., Atmospheric and Oceanic Fluid Dynamics, 2006 (Cambridge University Press).

von Helmholtz, H., Über Integrale der hydrodynamischen Gleichungen, welche der Wirbelbewegung entsprechen. J. für die reine und angewandte Mathematik, 1858, 55, 25-55.

Waugh, D., The efficiency of symmetric vortex merger. Phys. Fluids A, 1992, 4, 1745-1758.

Waugh, D. and Dritschel, D., The stability of filamentary vorticity in two- dimensional geophysical vortexdynamics models. J. Fluid Mech., 1991, 231, 575-598.

Williams, G., Planetary circulations: 1. Barotropic representation of Jovian and terrestrial turbulence. J. Atmos. Sci., 1978, 35, 1399-1424.

$\mathrm{Wu}, \mathrm{H}$. , Overman, E. and Zabusky, N., Steady-state solutions of the Euler equations in two dimensions: rotating and translating V-states with limiting cases. I. Numerical algorithms and results. J. Comput. Phys., 1984, 53, 42-71.

Yao, H., Dritschel, D. and Zabusky, N., High-gradient phenomena in two-dimensional vortex interactions. Phys. Fluids, 1995, 7, 539-548.

Yasuda, I. and Flierl, G., Two-dimensional asymmetric vortex merger: Contour dynamics experiment. J. Phys. Oceanogr., 1995, 51, 145-170.

Zabusky, N., Hughes, M. and Roberts, K., Contour Dynamics for the Euler Equations in Two Dimensions. J. Comput. Phys., 1979, 30, 96-106. 